\title{
CHARACTERIZATION AND SYNTHESIS OF RANDOM ACCELERATION VIBRATION SPECIFICATIONS
}

\author{
J.J. Wijker ${ }^{1}$, M.H.M. Ellenbroek ${ }^{2}$, and A. de Boer ${ }^{1}$ \\ ${ }^{1}$ University Twente \\ Drienerlolaan 5, 7522 NB Enschede, The Netherlands \\ e-mail: j.j.wijker@utwente.nl \\ ${ }^{2}$ Dutch Space, University Twente \\ Leiden, Enschede, The Netherlands \\ e-mail: m.ellenbroek@dutchspace.nl,a.deboer@utwente.nl
}

Keywords: Random vibrations, random vibration testing, random response analysis, damage response spectra.

\begin{abstract}
Random acceleration vibration specifications for subsystems, i.e. instruments, equipment, are most times based on measurement during acoustic noise tests on system level, i.e. a spacecraft and measured by accelerometers, placed in the neighborhood of the interface between spacecraft and subsystem. Tuned finite element models can be used to predict the random acceleration power spectral densities at other locations than available via the power spectral density measurements of the acceleration.

The measured and predicted power spectral densities do represent the modal response characteristics of the system and show many peaks and valleys.

The equivalent random acceleration vibration test specification is a smoothed, enveloped, peak-clipped version of the measured and predicted power spectral densities of the acceleration spectrum.
\end{abstract}

The original acceleration vibration spectrum can be characterized by a different number response spectra: Shock Response Spectrum (SRS), Extreme Response Spectrum (ERS), Vibration Response Spectrum (VRS), and Fatigue Damage Spectrum (FDS). An additional method of non-stationary random vibrations is based on the Rayleigh distribution of peaks. The response spectra represent the responses of series of SDOF systems excited at the base by random acceleration, both in time and frequency domain.

The synthesis of equivalent random acceleration vibration specifications can be done in a very structured manner and are more suitable than equivalent random acceleration vibration specifications obtained by simple enveloping. In the synthesis process Miles' equation plays a dominant role to invert the response spectra into equivalent random acceleration vibration spectra.

A procedure is proposed to reduce the number of data point in the response spectra curve by dividing the curve in a numbers of fields. The synthesis to an equivalent random acceleration 
spectrum is performed on a reduced selected set of data points. The recalculated response spectra curve envelops the original response spectra curves.

A real life measured random acceleration spectrum (PSD) with quite a number of peaks and valleys is taken to generate, applying response spectra SRS, ERS, VRS, FDS and the Rayleigh distribution of peaks, equivalent random acceleration vibration specifications. Computations are performed both in time and frequency domain. 


\section{INTRODUCTION}

Subsystems (instruments, equipment, boxes) are to be qualified against rather severe mechanical random acceleration vibration test specifications as discussed in [11]. The random acceleration vibration test specifications are, in general, enforced accelerations at the interface between spacecraft and subsystems. The random vibrations are mainly induced by the acoustic loads exposed to the spacecraft during launch and performing acoustic tests, representing the launch environment. The acoustic loads (sound pressures) are assumed to be diffuse and are simulated in a reverberant chamber, like the Large European Acoustic Facility (LEAF) at ESA/ESTEC, Noordwijk, The Netherlands.

The measured random accelerations and or similar predictions are broad-banded and shows many peaks as shown in Fig. 1 (blue line). These random acceleration measurements and predictions are converted into more or less equivalent smooth random acceleration vibration test specification, which represent as good as possible the underlying measured and calculated random acceleration responses. The equivalent random acceleration vibration test specification shall not lead to under-testing or significant over-testing of the test-item. An enveloping test random acceleration vibration specification is illustrated in Fig. 1 (black line), which is such severe that over-testing of the test item will certainly occur.

Several methods are available to reconstruct and characterize in a very structured manner the equivalent random acceleration vibration test specification from the measured and predicted random response data. The following characterization methods are discussed in this paper:

- Shock Response Spectrum (SRS) [19], Extreme Response Spectrum (ERS) [21]. Both response spectra are based on extreme responses of SDOF systems excited by enforced random accelerations, the first in the time and and the second in frequency domain.

- Vibration Response Spectrum (VRS) [16]. The VRS is the response spectrum based on the $3 \sigma$ responses of series of SDOFs, with varying natural frequency, excited at the base by random acceleration in the frequency domain.

- Fatigue Damage Spectrum (FDS) [21]. This damage response spectrum represents the cumulative damage due to the random responses of series of SDOF systems, with varying natural frequency, excited by random acceleration, both in the time and frequency domain.

Another method to synthesize the equivalent random vibration spectra is a method applied to pseudo stationary random vibration [8, 23], and is based on Rayleigh distribution of peaks (damage-potential) [36].

Miles' equation [24] is in the synthesis process of equivalent random acceleration vibration specifications from the different response spectra of great importance.

\section{PREVIOUS WORK}

The SRS was already mentioned by M.A. Biot in 1933 [4] and later in 1941 [5]. The theoretical description of the SRS was done within the frame of earthquake engineering. A mechanical analyzer was developed to predict stresses in structural systems under transient impulse. The concept of SRS of accelerations is nowadays still in use in spacecraft structure engineering to characterize the severity of high frequency transient accelerations, such as separation of launch vehicle stages, shroud and the separation of spacecraft [34]. In SVM-5 [19] the principles and methods to analyze shocks are discussed in depth. Smallwood developed in [30] a very 


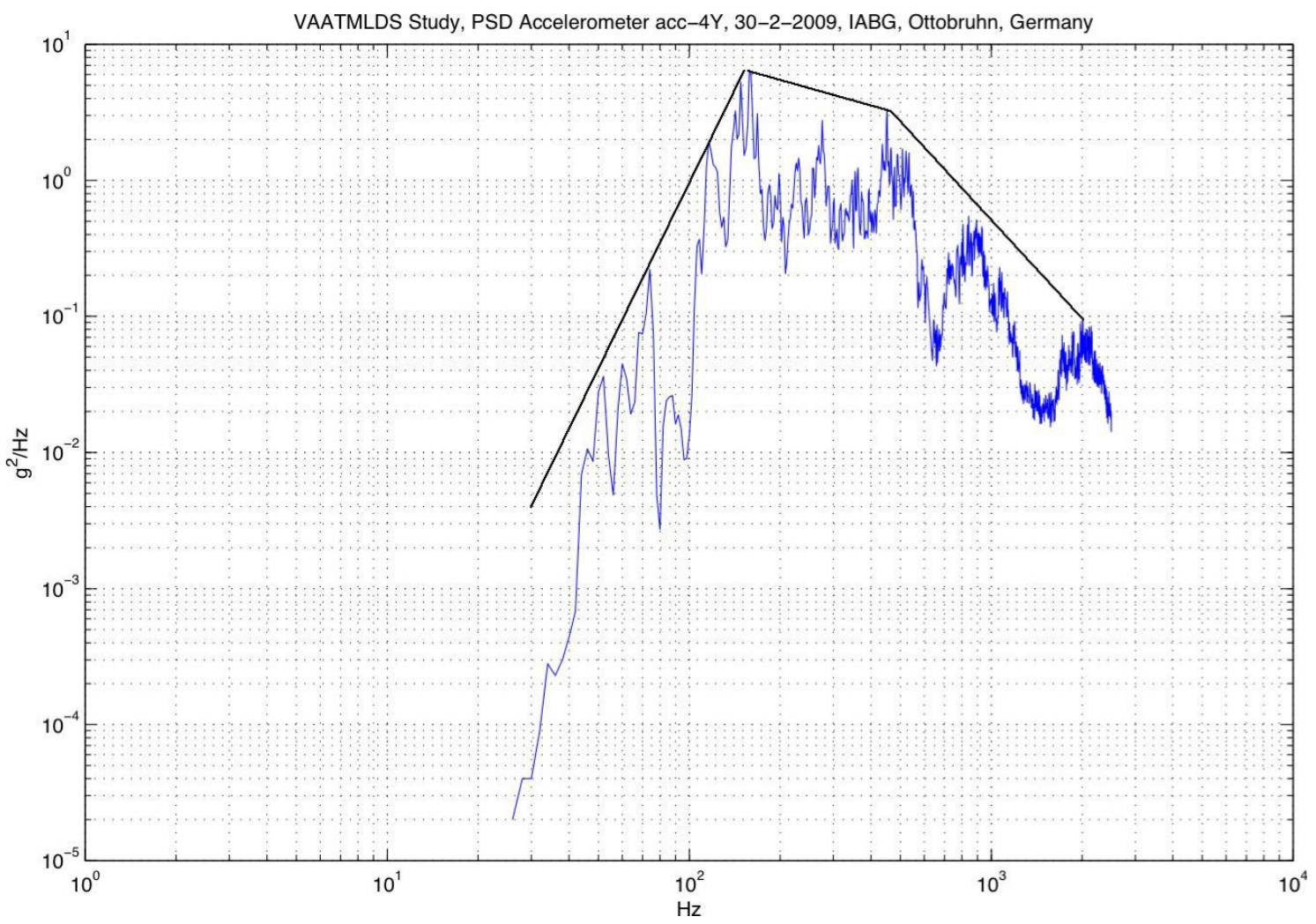

Figure 1: Schematic random acceleration vibration test specification (black) representing measured PSD responses (blue) $\mathrm{g}^{2} / \mathrm{Hz}$

efficient recursive formula to calculate the SRS. In [1, 32] the Smallwood recursive method (ztransform) was presented as a Matlab ${ }^{R}$ or Octave ${ }^{R}$ script. This script is applied to calculate the acceleration SRS in the time domain.

Lalanne discussed in his book "Specification Development" [21] the principle of the ERS and FDS for both sinusoidal and random vibration and the FDS for shocks as well.

Halfpenny et al discussed in their paper [13] the application of the SRS, ERS for peak accelerations and the FDS, to represent the cumulative fatigue damage caused by relative displacements (stresses), to describe new methods for vibration qualification of equipment on aircraft. Equivalent ERS and FDS are calculated for the proposed qualification random acceleration vibration test specification, which envelopes the flight ERS and SRS. The ERS is calculated in the frequency domain using numerical integration of the vibration spectrum and/or Miles' equation [24]. The peak values of stationary Gaussian process are discussed in detail in [27, 28]. In [13] the FDS is accurately obtained by numerical integration of the response spectrum or can be approximated applying Miles' equation. The approximate equation is used to calculate the equivalent random acceleration vibration spectrum.

Halfpennny described in his paper [12] the calculation of the the FDS of random vibrations both in the time and frequency domain. The random acceleration vibration spectrum is synthesized into the time domain by the summation of sine waves and the associated frequency, which in turn are applied to calculate the FDS in the time domain. The rain flow counting method is made available in Matlab ${ }^{\circledR}$ scripts, however, a number of rain flow counting methods are discussed in [3]. The synthesized random vibration test spectrum is calculated from the FDS, both in time and frequency domain, using the Miles' approximation for the FDS. The FDS is based 
on the relative displacements of the SDOF systems base excited by the random accelerations.

McNeil described in his paper [23] the application of the FDS, however, not based on the relative displacement, but on the relative pseudo-velocities of the SDOF systems. He stated that at resonance the pseudo-velocity is roughly proportional to the stress. The FDS is calculated both in the frequency and time domain. In the time domain rain flow counting is used to extract amplitudes and associated number of cycles. The equivalent random vibration spectrum is calculated inverting the FDS based on Miles' approximation. The second method discussed in this paper is based upon the Rayleigh distribution of peaks for non stationary random accelerations.

Eaton [10] described in his paper presented on the 2012 SCLV Conference a test tailoring methodology for equipment based on the ERS and FDS.

Dimaggio et al [8] mentioned the Rayleigh distribution of peaks a damage-based approach. The synthesis of the equivalent random vibration spectrum is again based on Miles' equation. Irvine presented on the 2012 SCLV conference a paper [18] a comparison of the damage potential method twith the SRS, ERS and VRS methods.

The VRS is described by Irvine in [16], which is used to synthesize random vibration spectra by enveloping the VRS. Miles' equation is applied to obtain synthesized random vibration spectra. The VRS spectrum is very similar to the ERS.

In the previous referenced papers (e.g. $[\overline{8}]$ ) it was recommended to vary the amplification factor and Basquin's exponent of the s-N curve, such that $Q=10,25,50$ and $b=4,8,12$. The worst synthesized random vibration spectra shall be applied to testing.

\section{RESPONSE SPECTRUM}

In this section a number of response spectra will be briefly discussed. The SRS is based on deterministic transient inputs and responses, while the VRS, ERS are based on the random PSD acceleration inputs and r.m.s. responses of SDOF systems. and the FDS is based on the response of SDOF systems both in the time and frequency domain. The random acceleration input is assumed to be weakly stationary and Gaussian. Mean values of responses are assumed to be zero.

\subsection{Shock Response Spectrum (SRS)}

The SRS was first introduced by Biot [4, 5] within the frame of earthquake engineering, and has been in existence for a long time.

The SRS is a graphical representation of an arbitrary transient acceleration input, how a single degree of freedom system (SDOF) (mass-spring-damper) responds to that input. Actually it shows the peak acceleration response in principle of an infinite number of SDOF systems, each of which has different natural frequencies $\left(\omega_{i}=2 \pi f_{i}, i=1,2, \cdots\right)$. This illustrated in Fig. 2, where the SDOF systems are mass normalized. In fact, the SRS analysis is the maximum response of a series of SDOF systems having the same damping to a given transient signal. In practice a damping ratio $\zeta=0.05(Q=10)$ is assumed.

The analysis procedure to compute the SRS is described in [1, 32], where a MATLAB ${ }^{\circledR}$ script has been presented based on the recursive formula proposed by Smallwood [30].

\subsection{Vibration Response Spectrum (VRS)}

Tom Irvine introduced the VRS in [16], which is similar to the SRS, however, the transient acceleration input is now replaced by random acceleration PSD input $W_{\ddot{u}}(f)\left(\mathrm{g}^{2} / \mathrm{Hz}\right)$ and the transient maximum responses are replaced by the $1 \sigma$ absolute responses of the SDOF systems. 


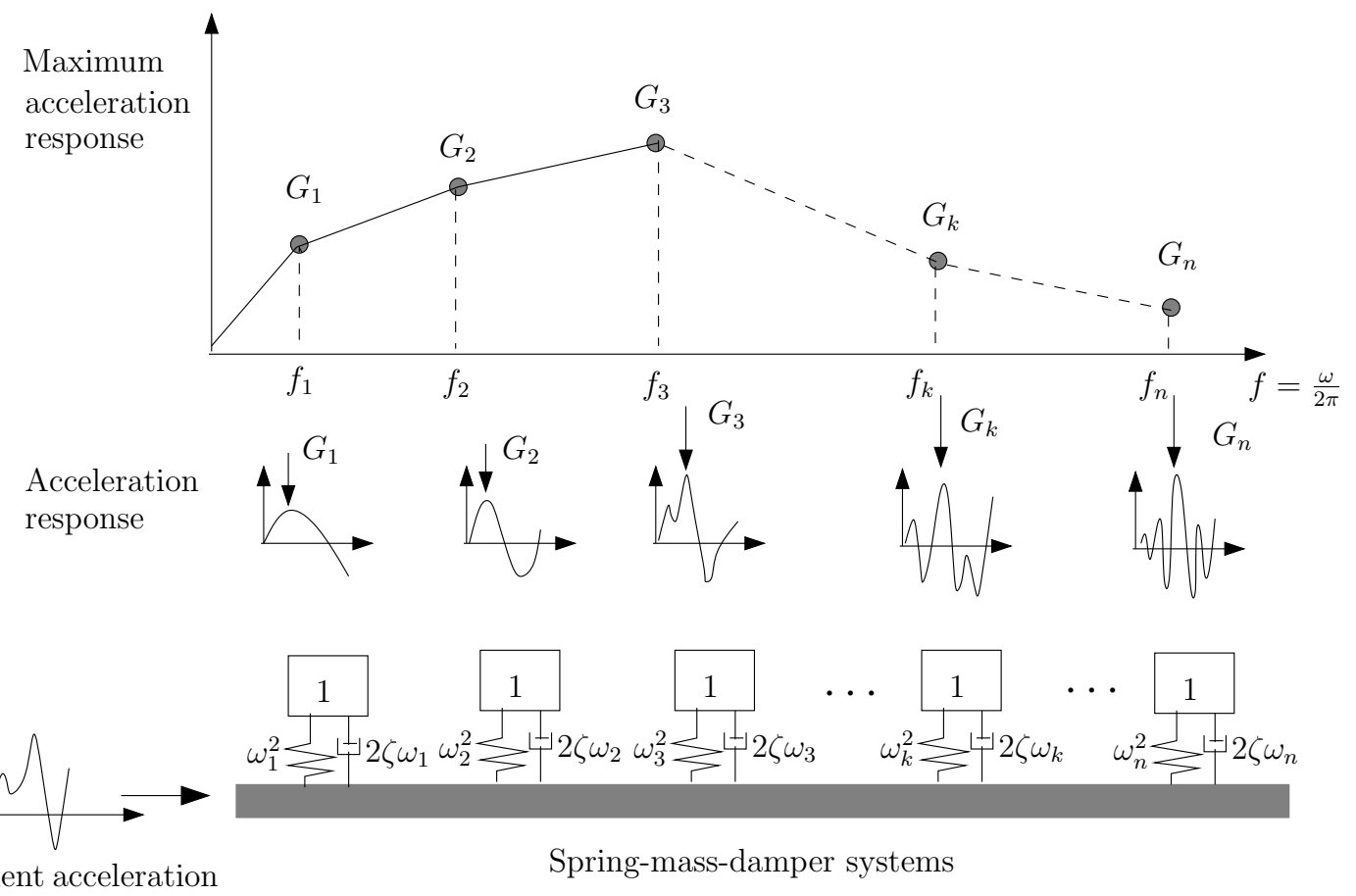

Figure 2: How a shock response spectrum is developed

In general, the damping ratio is taken $\zeta=0.05(Q=10)$ and is the same for all SDOF systems.

The graphical representation of the VRS is illustrated in Fig. 3. The SDOF systems are mass normalized. The standard deviation of the response of the SDOF system enforced at the base by random acceleration input can be calculated by the following expression

$$
\sigma_{i}=\sqrt{\int_{0}^{\infty}\left|H_{i}(f)\right|^{2} W_{\ddot{u}}(f) d f} \approx \sqrt{\sum_{f_{\min }}^{f_{\max }}\left|H_{i}(f)\right|^{2} W_{\ddot{u}}(f) \Delta f}, i=1,2, \cdots, N,
$$

where the frequency transfer function $(\mathrm{FRF}) H_{i}(f)$ is given by

$$
H_{i}(f)=\frac{1+2 j \zeta f / f_{i}}{1-\left(f / f_{i}\right)^{2}+2 j \zeta f / f_{i}},
$$

and $f_{i}=\omega_{i} / 2 \pi$ is the natural frequency.

If the input PSD $W_{\ddot{u}}(f)$ is relatively flat at frequencies near the natural frequency $f_{i}$ equation (15) can be approximated by Miles' equation [35]:

$$
\sigma_{i} \cong \sqrt{\frac{\pi f_{i} W_{\ddot{u}}\left(f_{i}\right)\left(1+4 \zeta^{2}\right)}{4 \zeta}} \approx \sqrt{\frac{\pi f_{i} Q W_{\ddot{u}}\left(f_{i}\right)}{2}}, i=1,2, \cdots, N .
$$

The VRS is defined as

$$
\operatorname{VRS}\left(f_{i}\right)=3 \sigma_{i}, i=1,2, \cdots, N .
$$

The VRS equivalent random acceleration vibration specification $W_{\ddot{u}}\left(f_{i}\right)$ for the enforced acceleration can be calculated by inverting of (3)

$$
W_{V R S, \ddot{u}}\left(f_{i}\right)=\frac{2(V R S)^{2}\left(f_{i}\right)}{9 \pi f_{i} Q}, i=1,2, \cdots, N .
$$



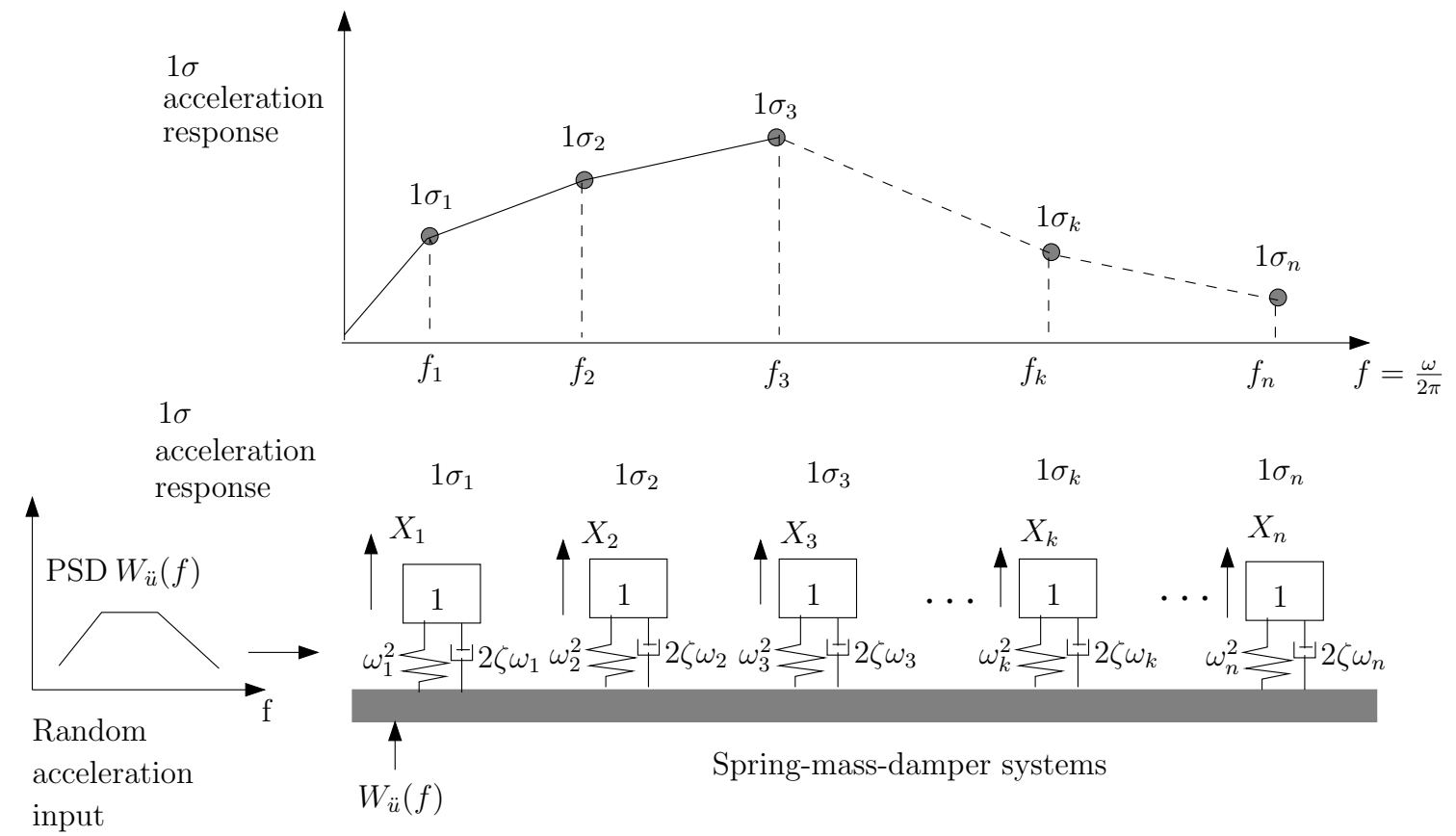

Figure 3: How a vibration response spectrum is developed

\subsection{Extreme (Peak) Response Spectrum (ERS)}

The ERS is similar to the SRS because peak values of the SDOF random response are depicted graphically in the ERS. Lalanne already discussed in [21] the ERS for random vibrations. The $1 \sigma_{i}$ response of the SDOF system " $i$ " is multiplied by a random peak factor $C_{i}$. The expected value of the peak factor of the random response of the SDOF system is given by [28]:

$$
E\left[C_{i}\right]=\sqrt{2 \ln \left(f_{i} T\right)}+\frac{\gamma}{\sqrt{2 \ln \left(f_{i} T\right)}},
$$

and the standard deviation of the peak factor is

$$
\sigma\left[C_{i}\right]=\frac{\pi}{\sqrt{6}} \frac{1}{\sqrt{2 \ln \left(f_{i} T\right)}}
$$

where $f_{i}$ is the natural frequency of the SDOF system, $T$ the time duration of the random accelerations process and $\gamma=0.5772$ is the Euler constant [9]. Further it is assumed that $f_{i} T \gg 1$, and

- the random response $X_{i}$ of the SDOF system has a Gaussian distribution, and

- the peak values of the random response of the SDOF system are statically independent. The statistically independence assumption is acceptable if $E\left[C_{1}\right] / \sigma\left[C_{1}\right]>3.5$ and $f_{i} T>$ 250 for a Gaussian process [14].

With a given standard deviation $\sigma_{i}$ of the random response of the SDOF system the maximum expected extreme (peak) acceleration response spectrum $E R S_{a}\left(f_{i}\right)$ can be calculated using:

$$
E R S_{a}\left(f_{i}\right)=E\left[C_{i}\right] * \sigma_{i}, i=1,2, \cdots, N .
$$


The expected peak values for the displacement, the $E R S_{d}\left(f_{i}\right)$ displacement spectrum, is given by [13]

$$
E R S_{d}\left(f_{i}\right)=9.81^{2} \frac{E\left[C_{i}\right] * \sigma_{i}}{\omega_{i}^{2}}, i=1,2, \cdots, N
$$

in case the PSD input spectrum $W_{\ddot{u}}(f)$ is specified in $\mathrm{g}^{2} / \mathrm{Hz}$.

The ERS equivalent random acceleration vibration specification $W_{E R S, \ddot{u}}\left(f_{i}\right)$ for the enforced acceleration can be calculated with the aid of inverting (8)

$$
W_{E R S, \ddot{u}}\left(f_{i}\right)=\frac{2 E R S^{2}\left(f_{i}\right)}{E\left(C_{i}\right)^{2} \pi f_{i} Q}, i=1,2, \cdots, N .
$$

Because the SRS is also based on peak responses, the equivalent random acceleration vibration specification can be obtained using (10),

$$
W_{S R S, u}\left(f_{i}\right)=\frac{2 S R S^{2}\left(f_{i}\right)}{E\left(C_{i}\right)^{2} \pi f_{i} Q}, i=1,2, \cdots, N .
$$

\subsection{Fatigue Damage Spectrum (FDS)}

In this section the fatigue damage spectrum will be based on the relative displacements FDS $_{d}$ and pseudo-velocities [23] FDS $_{p v}$.

\subsubsection{FDS $_{d}$ in Frequency Domain}

The expected cumulative fatigue damage $E[D(T)]$ (in one SDOF system) involves the PalgrenMiner fatigue accumulation rule [20, 22] in combination of the s-N curve $N_{k} s_{k}^{b}=C\left(N_{k}\right.$ is the number of allowable cycles at stress level $s_{k}$ ) for a narrow-banded Gaussian process the cumulative damage and is given by [7, 36] for $f_{1}, i=1,2, \cdots N$ :

$$
E\left[D\left(T, f_{i}\right)\right]=\frac{f_{i} T}{C}\left(\sqrt{2} \sigma_{s}\right)^{b} \Gamma\left(1+\frac{b}{2}\right)
$$

where $C$ is a material constant (Basquin coefficient), $b$ is the fatigue exponent (Basquin's exponent), $f_{i}$ is the natural frequency of the SDOF system, $T$ the time duration, $\sigma_{s}$ is the standard deviation of the stress, and $\Gamma$ is the gamma function.

The stress $s$ is proportional to the relative displacement of the SDOF multiplied by a constant $K$. The standard deviation of the stress $\sigma_{s, i}$ can be obtained as follows:

$$
\sigma_{s, i}=K \frac{9.81}{\left(2 \pi f_{i}\right)^{2}} \sqrt{\frac{\pi}{2} f_{i} Q W_{\ddot{u}}\left(f_{i}\right)}
$$

because the PSD spectrum $W_{\ddot{u}}$ of the enforced random acceleration is most times specified in $\mathrm{g}^{2} / \mathrm{Hz}$.

The $\mathrm{FDS}_{d}$ corresponding to the natural frequency of the SDOF system " $i$ " is now defined for $f_{i}, i=1,2, \cdots N$ :

$$
F D S_{d}\left(T, f_{i}\right)=f_{i} T \frac{K^{b}}{C}\left[\frac{9.81^{2} Q W_{\ddot{u}}\left(f_{i}\right)}{2\left(2 \pi f_{i}\right)^{3}}\right]^{\frac{b}{2}} \Gamma\left(1+\frac{b}{2}\right) .
$$

In general, the constants are taken as $K=C=1$, the exponent $b=4,8,12$, and the amplification factor $Q=10,25,50$. 
Instead of using Miles' equation, the standard deviation of the relative displacement response $\sigma_{d, i}$ of the SDOF system can be computed as follows

$$
\sigma_{d, i}=\sqrt{\int_{0}^{\infty}\left|H_{d, i}(f)\right|^{2} W_{\ddot{u}}(f) d f} \approx \sqrt{\sum_{f_{\min }}^{f_{\max }}\left|H_{d, i}(f)\right|^{2} W_{\ddot{u}}(f) \Delta f}, i=1,2, \cdots, n,
$$

where the frequency transfer function $(\mathrm{FRF}) H_{i}(f)$ is given by

$$
H_{d, i}(f)=\frac{1}{\left(2 \pi f_{i}\right)^{2}} \frac{1}{\left(1-\left(f / f_{i}\right)^{2}+2 j \zeta f / f_{i}\right)} .
$$

The standard deviation of the stress $s_{i}$ is $\sigma_{s_{i}}=9.81 K \sigma_{d, i}$. The mean value of the stress is assumed to be zero.

The synthesized equivalent random acceleration vibration spectrum $W_{F D S_{d}, \ddot{u}}\left(f_{i}\right)$ can be calculated by the inverse of 14

$$
W_{F D S_{d}, \ddot{u}}\left(f_{i}\right)=\frac{2\left(2 \pi f_{i}\right)^{3}}{9.81^{2} Q}\left[\frac{C F D S_{d}\left(f_{i}\right)}{K^{b} f_{i} T \Gamma\left(1+\frac{b}{2}\right)}\right]^{\frac{2}{b}}, i=1,2, \cdots, N .
$$

\subsubsection{FDS $_{d}$ in Time Domain}

If the random acceleration is provided in the time domain or is synthesized into the time domain, the the cumulative damage $D$ is given by the Palgren Miner rule, which tells us that cumulative damage $D$ is

$$
D=\sum_{k=1}^{N} \frac{n_{k}}{N_{k}}
$$

where $n_{k}$ is the number of stress oscillations at stress level $s_{k}$, and $N_{k}$ is the number of allowable oscillation at stress level $s_{k}$ given by the $s-N$ curve

$$
s_{k}^{b} N\left(s_{k}\right)=C \text {. }
$$

The generation of the $\mathrm{FDS}_{d}$ in the time domain is symbolically illustrated in Fig. 4 . The random enforced transient acceleration is applied to the base of each of the SDOF systems. From the calculated random relative displacements $z(t)$, per SDOF system, the numbers of cycles and associated "stress" peaks are extracted using a rain flow counting procedure [2, 26]. Using "stress" peaks and cycles the fatigue damage spectrum can be obtained. Again the stress constant and the Basquin's coefficient are assumed to be $K=C=1$ and the Basquin exponent may vary $b=4,8,12$.

\subsubsection{FDS $_{p v}$ in Frequency Domain (Spectral Method)}

in [23] the pseudo-velocity $p v(t)$ of the SDOF system is applied to calculate the $\operatorname{FDS}_{p v}$, because the pseudo-velocity is roughly proportional to stress for many structures, a scale factor exists between the stress $\sigma$ and the pseudo-velocity $p v, \sigma=K p v$, [6, 17]. The maximum strain amplitude $\epsilon$ is proportional to the "vibration Mach number" $v / c$, where $v$ is the maximum 


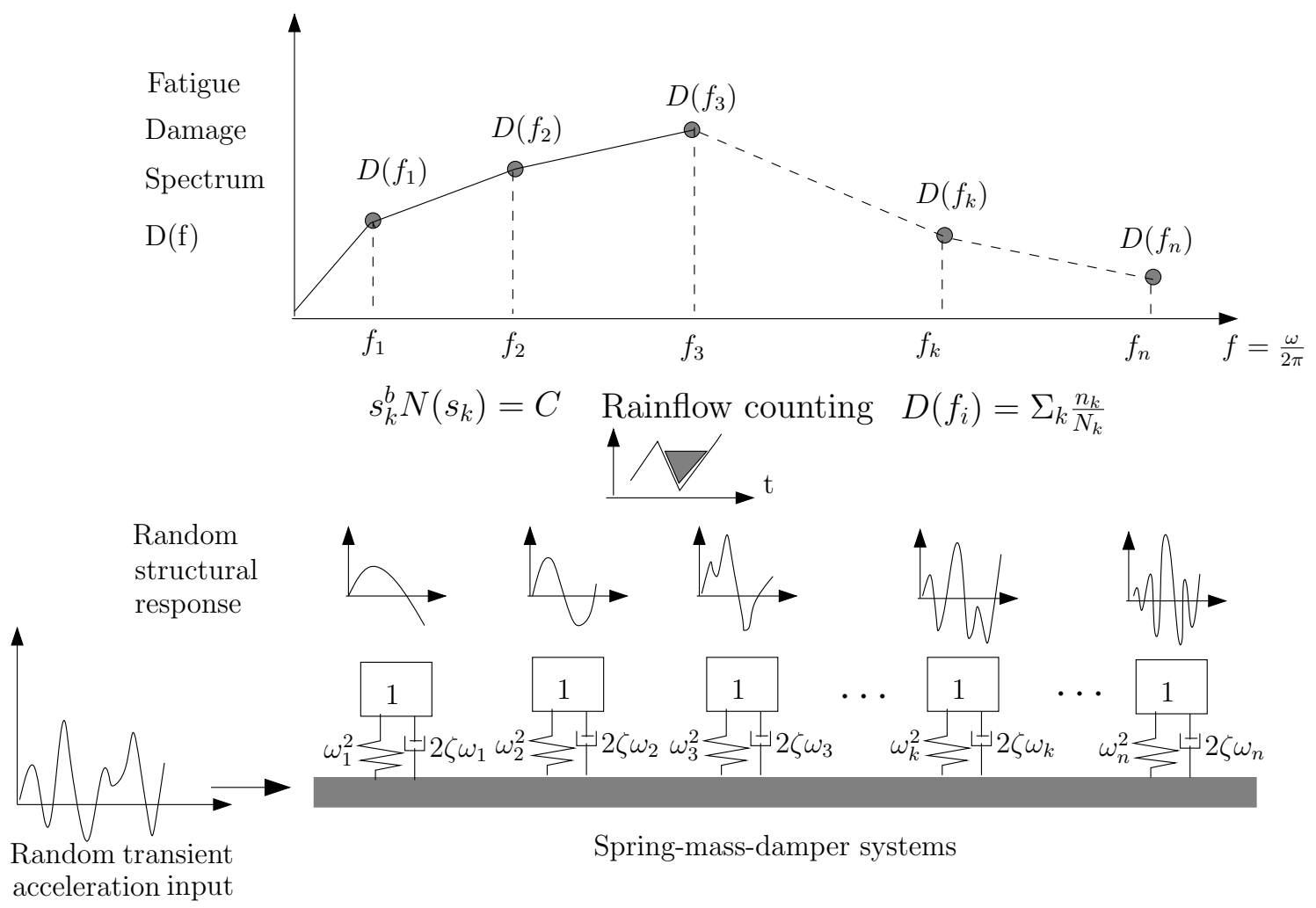

Figure 4: How a time domain damage fatigue spectrum is developed

vibratory velocity and $c$ is the speed of sound in the material. The maximum strain is given by $\epsilon=k(v / c)$, where $k=0.145-2.00$ for many different configurations [6].

The pseudo-velocity is defined as the relative displacement response $z(t)$ of the SDOF system multiplied by the natural frequency of that SDOF, $p v(t)=z(t) *\left(2 \pi f_{i}\right)$.

The expected cumulative fatigue damage $E[D(T)]$ involves the Palgren-Miner fatigue accumulation rule [22] in combination of the s-N curve $N_{k} s_{k}^{b}=C\left(N_{k}\right.$ is the number of allowable cycles at stress level $s_{k}$ ) for a narrow-banded Gaussian process the expected cumulative damage of FDS and is given by [7, 36], for $f_{1}, i=1,2, \cdots N$

$$
F D S_{p v}\left(f_{i}\right)=E\left[D\left(T, f_{i}\right)\right]=\frac{f_{i} T}{C}\left(\sqrt{2} \sigma_{s}\right)^{b} \Gamma\left(1+\frac{b}{2}\right)=\frac{f_{i} T}{C} K^{b}\left(2 \sigma_{p v}^{2}\right)^{\frac{b}{2}} \Gamma\left(1+\frac{b}{2}\right) .
$$

The standard deviation of pseudo-velocity $\sigma_{p v, i}$ is expressed as follows:

$$
\sigma_{p v, i}=9.81 \sqrt{\frac{1}{8 \pi f_{i}} Q W_{\ddot{u}}\left(f_{i}\right)},
$$

where the PSD function $W_{\ddot{u}}$ is most times given in $\mathrm{g}^{2} / \mathrm{Hz}$.

In general, the constants are taken as $K=C=1$, the exponent $b=4,8,12$, and the amplification factor $Q=10,25,50$.

Instead of using Miles' equation, the standard deviation of the pseudo-velocity response $\sigma_{p v, i}$ of the SDOF system can be numerically calculated as follows

$$
\sigma_{p v, i}=9.81 \sqrt{\int_{0}^{\infty}\left|H_{p v, i}(f)\right|^{2} W_{\ddot{u}}(f) d f} \approx \sqrt{\sum_{f_{\min }}^{f_{\max }}\left|H_{p v, i}(f)\right|^{2} W_{\ddot{u}}(f) \Delta f}, i=1,2, \cdots, n,
$$


where the frequency transfer function (FRF) $H_{p v, i}(f)$ is given by

$$
H_{p v, i}(f)=\frac{1}{\left(2 \pi f_{i}\right)} \frac{1}{\left(1-\left(f / f_{i}\right)^{2}+2 j \zeta f / f_{i}\right)} .
$$

The standard deviation of the pseudo-velocity $s_{i}$ is $\sigma_{s_{i}}=K \sigma_{p v, i}$. The mean value of the stress is zero.

The PSD values $W_{F D S_{p v} \ddot{u}}\left(f_{i}\right), i=1,2, \cdots, N$ of the random acceleration vibration specification can be obtained inverting (20) in combination with (21). Thus we get

$$
\sigma_{p v}^{2}\left(f_{i}\right)=\frac{1}{2}\left(\frac{C F D S_{p v}\left(f_{i}\right)}{f_{i} T K^{b} \Gamma\left(1+\frac{b}{2}\right.}\right)^{\frac{2}{b}},
$$

and

$$
W_{F D S_{p v} \ddot{u}}\left(f_{i}\right)=\frac{8 \pi f_{i} \sigma_{p v}^{2}\left(f_{i}\right)}{9.81^{2} Q}, i=1,2, \cdots, N
$$

\subsubsection{FDS $_{p v}$ in Time Domain}

The calculation of $\mathrm{FDS}_{p v}$ is identical to calculation of the $\mathrm{FDS}_{d}$, however, the stress is now proportional to the pseudo-velocity. If the random pseudo-velocity is provided in the time domain the the cumulative damage $D$ is given by the Palgren Miner rule, which tells us that cumulative damage $D$ is

$$
D=\sum_{k=1}^{N} \frac{n_{k}}{N_{k}}=\frac{K^{b}}{C} \sum_{k=1}^{N} n_{k} p v_{k}^{b},
$$

where $n_{k}$ is the number oscillations at pseudo-velocity level $p v_{k}$, and $N_{k}$ is the number of allowable oscillation at pseudo-level level $p v_{k}$ given by the $s-N$ curve

$$
s_{k}^{b} N\left(s_{k}\right)=K^{b} p v^{b} N(p v)=C .
$$

The constants are taken $K=C=1$ and, in general, the exponent $b=4,8,12$.

The calculation procedure for $\mathrm{FDS}_{p v}$ is the similar to the calculation of $\mathrm{FDS}_{d}$ as shown in Fig. 4.

\subsection{Pseudo Stationary Random Vibration, Damage Potential}

The method of pseudo stationary random vibration described in this section was discussed in [8, 23], however, McNeil applied this method using pseudo velocities, as discussed in sections 3.4 .3 and 3.4.4

The acceleration response of a lightly damped SDOF system excited by a zero-mean, stationary Gaussian white noise excitation is narrow banded and the probability of peak values is a Rayleigh distribution function and given by

$$
f(A)=\left(\frac{A}{\sigma^{2}}\right) \exp \left(\frac{-A^{2}}{2 \sigma^{2}}\right), A \geq 0,
$$

where $A$ is the amplitude and $\sigma$ is the standard deviation. For a zero-mean response the standard deviation is equal to the r.m.s. value of the response. The probability the amplitude is grater then $A, A>$ can be obtained by integrating the probability density function from $A$ to $\infty$

$$
P(A>)=\int_{A}^{\infty} f(a) d a=\exp \left(\frac{-A^{2}}{2 \sigma^{2}}\right) .
$$


If we consider a stationary test of duration $T_{o}$, the total time $T(A>)$, or cumulative duration, spent during which response cycles with amplitudes exceeding $A$ occur is

$$
T(A>)=T_{o} \exp \left(\frac{-A^{2}}{2 \sigma^{2}}\right) .
$$

For a SDOF system, with natural frequency $f_{i}$, the mean square acceleration response to a broad-band Gaussian excitation with a power spectral density $W\left(\mathrm{~g}^{2} / \mathrm{Hz}\right)$ is approximated by Miles' equation

$$
\sigma^{2}=\frac{\pi}{2} f_{i} Q W\left(f_{i}\right)
$$

Taking the natural logarithme of both sides of (30) and substituting (31) yields

$$
\ln \left(\frac{T_{o}}{T(A>)}\right)=\frac{1}{\pi f_{i} W\left(f_{i}\right)} \frac{A^{2}}{Q} .
$$

For a particular natural frequency $f_{i}$, the function (32) appears as a straight line on a plot of $\ln T(A>)$ versus linear $A^{2} / Q$ as shown in Fig. 5. In Fig. 5, $A_{\max }$ is the expected maximum amplitude at the exceedence duration of one period $1 / f_{i}$. Prescribing a test based on an en-

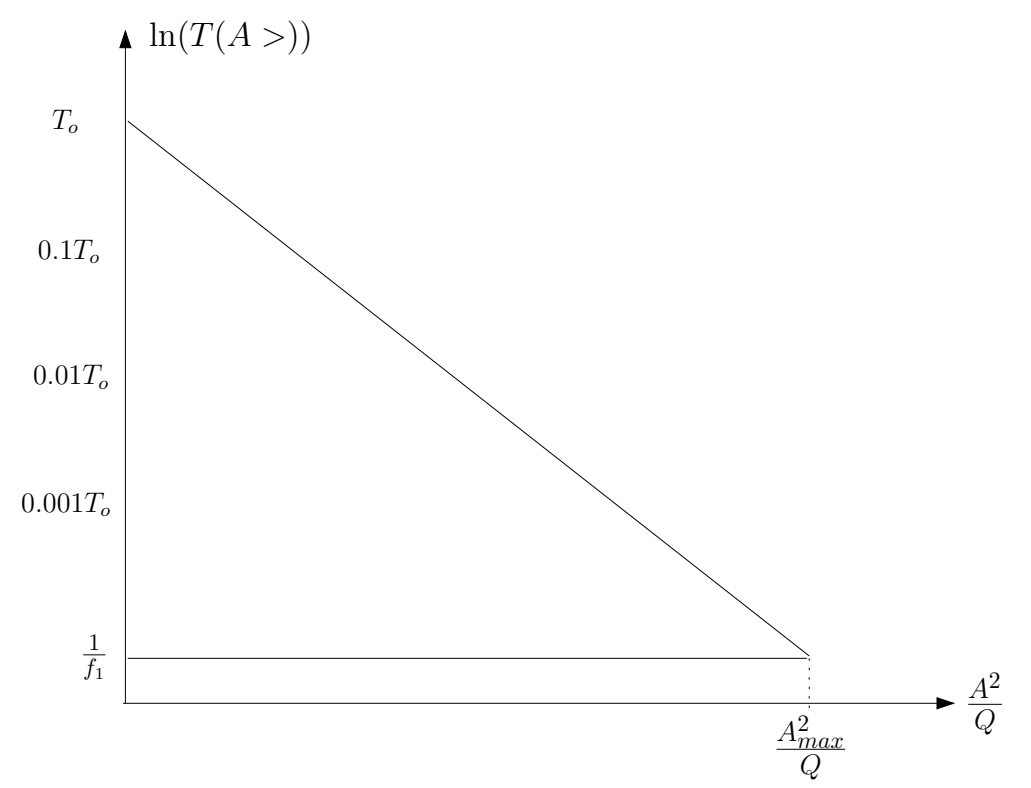

Figure 5: Cumulative duration plot for Rayleigh distributed Maxima

veloping Rayleigh line guarantees that the damage potential of the test, for a resonance of that frequency, envelops the damage potential of flight loads over the range of damping uncertainty. The Rayleigh line starts at $T_{o}$ and $A^{2} / Q=0$ and goes to a minimum time, the duration of the period $1 / f_{i}$, associated with $A_{\text {max }}^{2} / Q$. The total time $T_{o}=n_{A_{\min }>} / f_{i}$, where $n_{A_{\min }>}$ the number of cycles with amplitudes $A \geq A_{\min >}$. The number of cycles can be obtained by the rain flow counting method. The equivalent power spectral density $W_{D P, \ddot{u}}\left(f_{i}\right)$ corresponding to the the line $T_{o}$ to $A_{\max }^{2} / Q$ can be calculating by the following expression

$$
W_{D P, \ddot{u}}\left(f_{i}\right)=\frac{\left(A_{\max }^{2} / Q\right)}{\pi f_{i} \ln \left(f_{i} T_{o}\right)} .
$$

To construct the Rayleigh line (Fig. 5) the following step by step procedure is to be done: 
1. Translate the power spectral density spectra of the random vibration into a time domain random excitation.

2. Determine the absolute random acceleration response of the SDOF system, with natural frequency $f_{1}$, excited by the random excitation in the time domain.

3. Perform a rain flow counting to analyze the spectrum of amplitudes and corresponding number of cycles

4. Select a number of amplitudes $A_{k}, k=1,2, \cdots$, analyze the number of cycles $n\left(A_{k}>\right.$ ), $k=1,2, \cdots$ and calculate the durations $T\left(A_{k}>\right)=n\left(A_{k}>\right) / f_{i}, k=1,2, \cdots$. The minimum number of cycles beyond $A_{\max }$ is $n\left(A_{\max }>\right)=1$, with $T\left(A_{\min }>\right)=1 / f_{i}$, and the number of cycles beyond $A_{\min }$ is $n\left(A_{\min }>=T_{o} / f_{i}\right.$. This step is illustrated in Fig. 6.

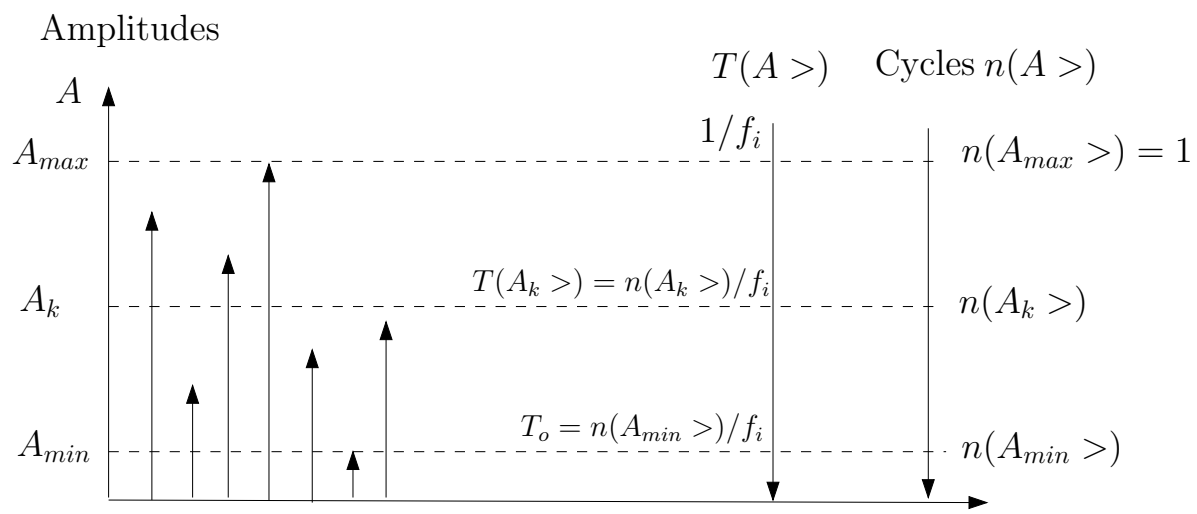

Figure 6: The evaluation of $T(A>)$ versus the amplitude $A$

\section{SYNTHESIS OF EQUIVALENT RANDOM ACCELERATION VIBRATION SPEC- IFICATION}

It is assumed that the equivalent random acceleration vibration spectra represent the same amount of (fatigue) damage as expected for the original measured or calculated spectrum. The reconstruction or synthesis of an equivalent random acceleration vibration specification $W_{\ddot{u}}(f)$ can be done using the response spectra; SRS, VRS, ERS, FDS ${ }_{d}, \mathrm{FDS}_{p v}$ and the Rayleigh line (damage potential). The response spectra computing in the time domain can be used as well. For that purpose the equations (5), (10), (11), (17), (25), and (33) can be applied. Miles' equation has a key role computing the equivalent random acceleration specifications. The procedure to reconstruct the equivalent random acceleration vibration specification is illustrated in Fig. 7 ( $\mathrm{a}$ and $\mathrm{b}$ ). In order to address the variations in damping and $\mathrm{s}-\mathrm{N}$ curve fatigue exponent $b$, the response spectra and Rayleigh line shall be computed with amplification factor values of $Q=10,25,50$ and exponents $b=4,8,12$ for each natural frequency $f_{i}$ of the SDOF systems. The greatest power spectral density value of $W_{\ddot{u}}\left(f_{i}\right)$ over the 3-9 variations of $Q$ and $b$ is used as the random equivalent vibration specification level at frequency $f=f_{i}$. Finally, the complete random acceleration vibration specification is established. However, engineering judgement is still needed. 


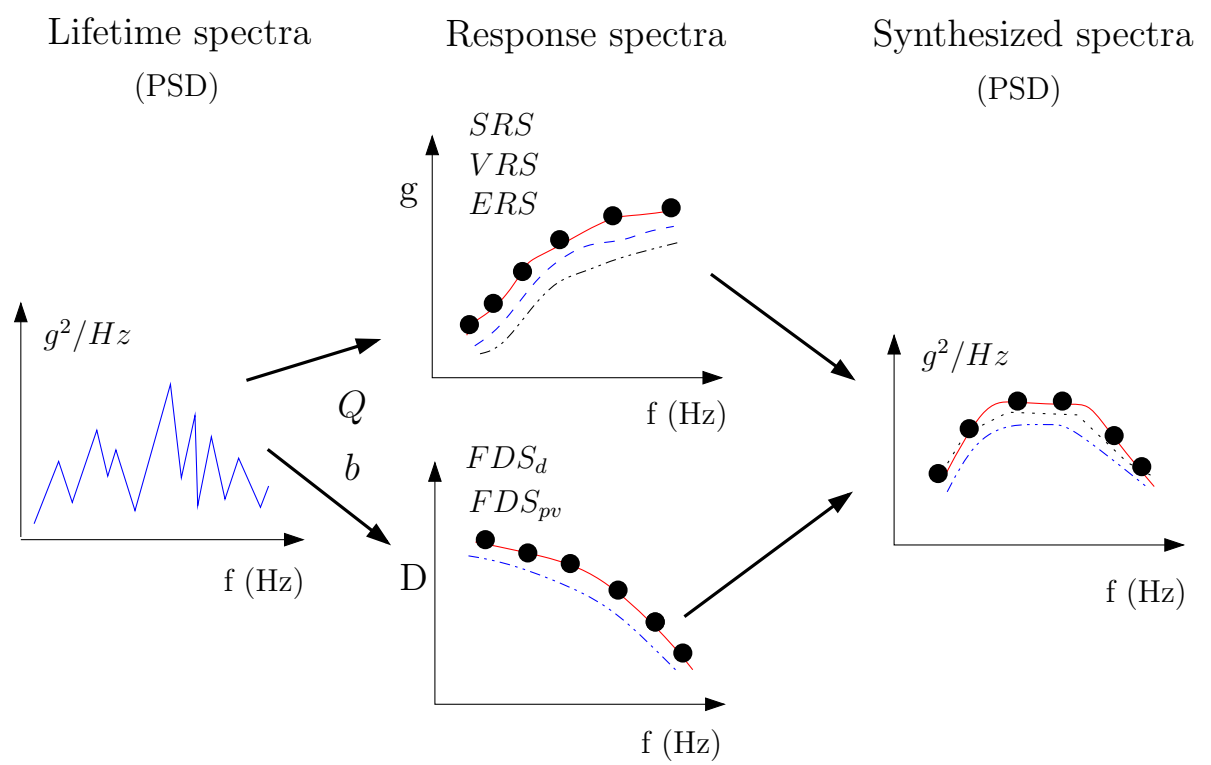

(a)

Random excitation Rayleigh line Random vibration specification time domain

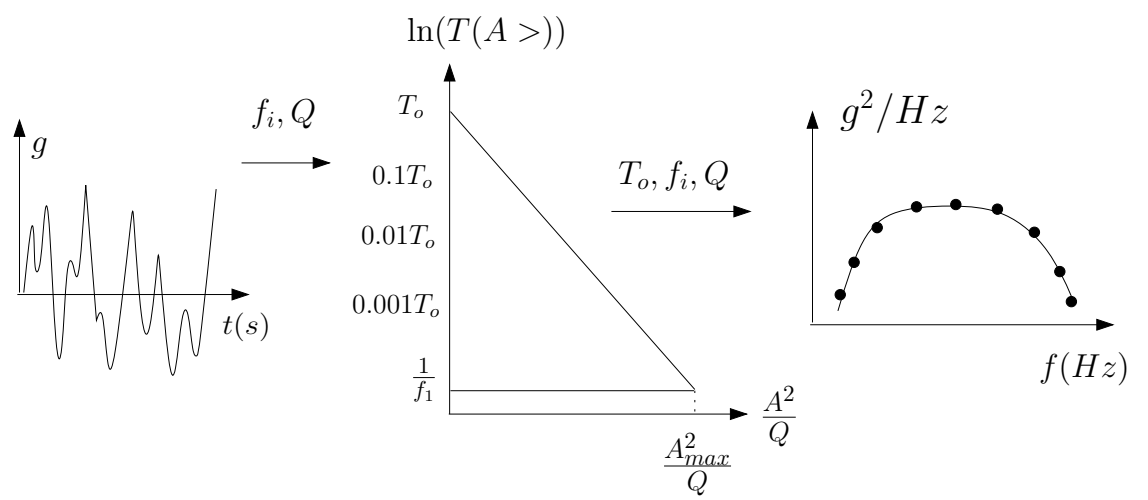

(b)

Figure 7: Schemes to synthesize the random acceleration vibration specification

\section{APPLICATION}

\subsection{Random Vibration Measurement during a Acoustic Test}

Within the frame of the ESA/TRP study: "Vibro-Acoustic Analysis Test methods for Large Deployable Structures" (VAATMLDS) [33] acoustic tests were performed on the VAATMLDS Bread Board solar array wing in the acoustic reverberant chamber of IABG [15].

The read-out of accelerometer Acc-4Y (Fig. 9) is the random acceleration spectrum, for which a smooth and shaker controllable random vibration acceleration test spectrum should be generated, as illustrated in Fig. 1. The read-out of accelerometer Acc-4Y is in fact the raw random acceleration vibration specification. The number of data points is 1251 and the frequency increment $\Delta f=2 \mathrm{~Hz}$. The generation of the random acceleration vibration test specification will be performed in a more or less structured manner applying the response spectra; SRS, ERS, VRS, FDS and the Rayleigh distribution of peaks. To account for uncertainties in damp- 


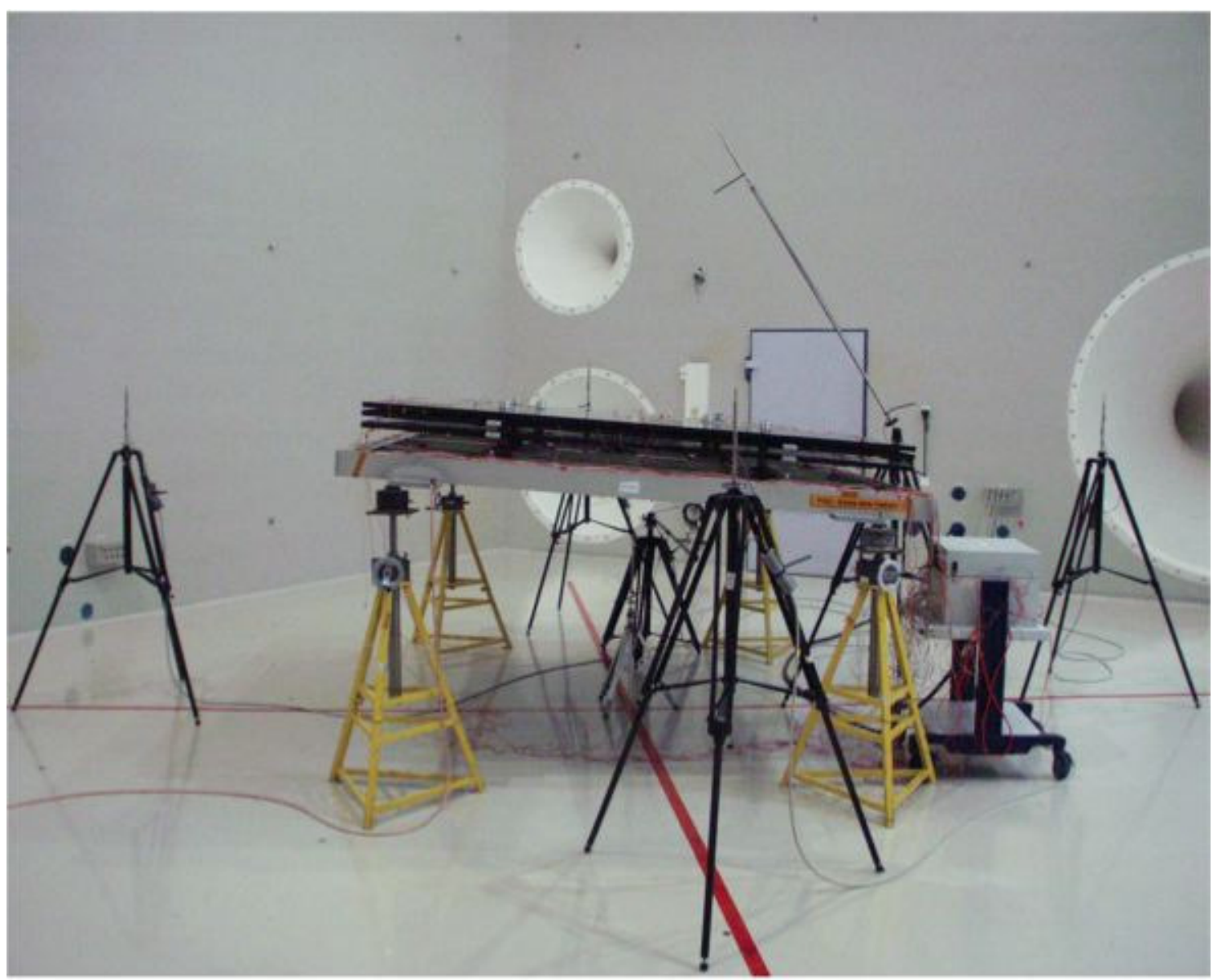

Figure 8: VAATMLDS bread board 3 panel solar array wing in IABG acoustic chamber

ing and Palgren-Miner cumulative damage rule, the amplification factor (quality factor) will be varied $Q=10,25,50$ and fatigue exponent $b=4,8,12$. Matlab ${ }^{\circledR}$ is applied to perform all computations.

The random acceleration spectrum is synthesized into the time domain (waveform) using the method described in [29], in combination with the Fourier transform [25]. The synthesized equivalent signals in the time domain are shown Fig. 10. It should be noticed that the synthesized time domain accelerations are random and will vary from analysis to analysis, however, having the same mean and standard deviation. This is due to the random frequency shifts in the arguments of the sine waves. The time increment $\delta t=1 / 2 f_{\max }=2.0 \times 10^{-4} \mathrm{~s}$, and the total time $T=2 N \delta t=0.5004 \mathrm{~s}$. The mean value of the all time domain signals is $\mu=0$, and the standard deviation $1 \sigma=$ r.m.s. $=24.98 \mathrm{~g}$. In case the time domain random acceleration is a Gaussian process the skewness $\lambda=0$ and the kurtosis $\gamma=3$ [31]. A skewness $\gamma>3$ will result in higher peaks and a skewness $\gamma<3$ will result in lower peaks compared to the ideal Gaussian process.

The measured accelerations of accelerometer Acc-4Y, during the acoustic test, are now available both in the frequency and time domain. The response spectra: SRS, ERS, VRS, FDS $_{d}$, $\mathrm{FDS}_{p v}$ and the distribution of Rayleigh peaks (damage potential) can now be computed.

The response spectra will be divided into $N_{\text {field }}$ fields containing each $n_{\text {field }}$ data points. This is illustrated in Fig. 11. The parameter $N_{\text {field }}$ is set to $N_{\text {field }}=100$. In each field 


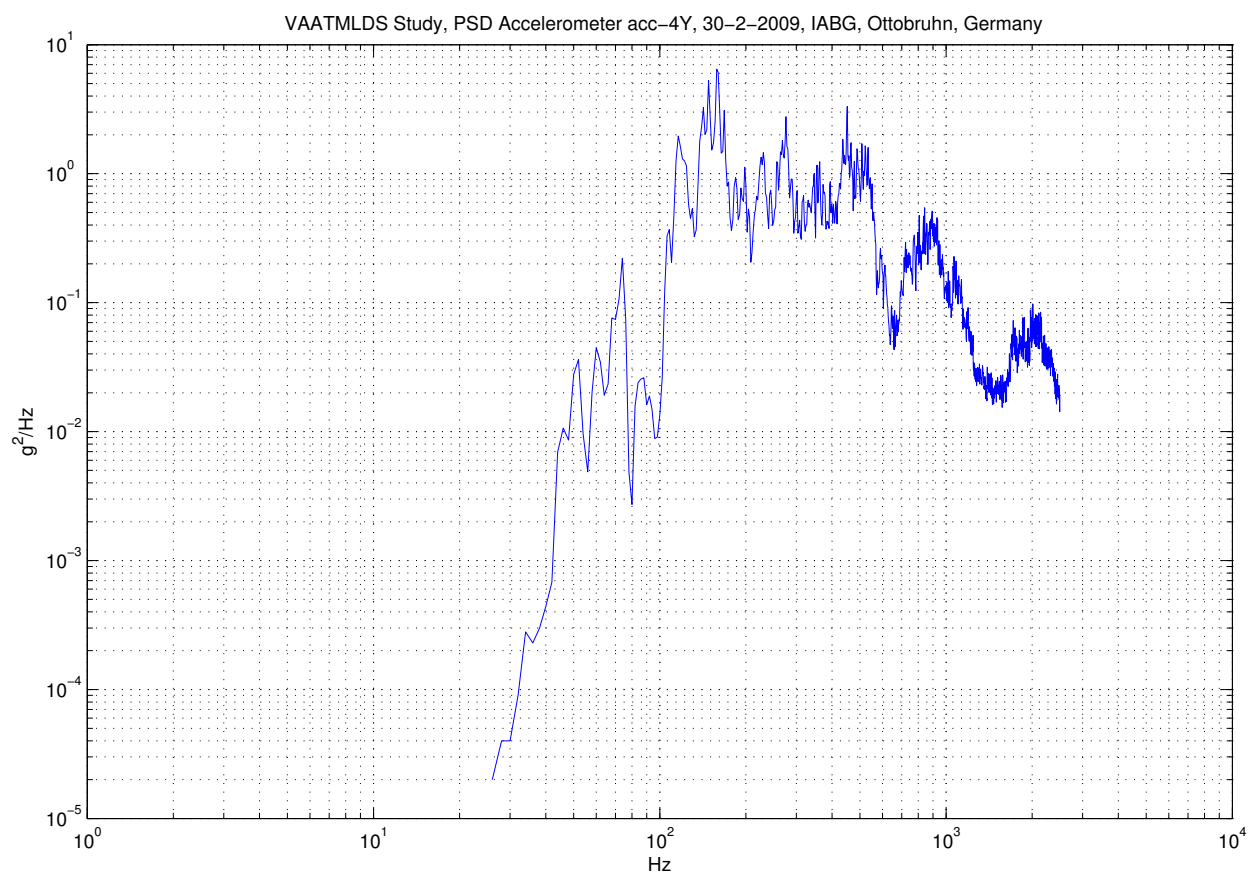

Figure 9: PSD measurement of accelerometer 4-Y, Grms=24.98g

the maximum value of the spectrum, and corresponding frequency, is detected and used to calculate the synthesized PSD of the random acceleration vibration specification. The local maximum may be an internal point or at the boundaries of the field. The first and last data point of the complete spectrum are considered too. The synthesized PSD's at the detected points are connected to each other assuming the log-log scales for the spectra and corresponding frequencies, respectively. Internal PSD values can be obtained by the following expression

$$
N=\log \left(\frac{W_{2}}{W_{1}}\right) / \log \left(\frac{f_{2}}{f_{1}}\right), W_{i}=W_{1}\left(\frac{f_{i}}{f_{1}}\right)^{N}=W_{1}\left(\frac{f_{i}}{f_{1}}\right)^{\frac{m}{3}},
$$

where $W_{1}$ and $W_{2}$ are the PSD values of the extreme points, $f_{1}$ and $f_{2}$ are the associated frequencies and $m$ is the slope of the spectrum in $\mathrm{dB} /$ octave. Index $i$ indicates a point with frequency $f_{i}$ in between the extreme points. Equation (34) is applied to calculate the area (mean square) under the synthesized equivalent PSD spectrum.

\subsection{Synthesis of Random Acceleration Vibration Specification from SRS and ERS}

In this section the synthesized equivalent random acceleration vibration specification is calculated based on the SRS and ERS spectra. The SRS and ERS have 1251 and 2481 data points, respectively. Both are ranging from $20-2500 \mathrm{~Hz}$. The SRS and ERS are computed in accordance to the methods mentioned in sections 3.1 and 3.3. The VRS is common to the ERS, and therefor not considered. The computed ERS and SRS are shown Fig. 12. (a).

The SRS and ERS overlay each other very well. Instead of computing the SRS in the time domain the ERS is a very good representative shock spectrum. Most times in spacecraft structures engineering the $3 \sigma$ VRS spectrum is used, however, it is recommended to use the ERS as the SRS for a given random acceleration vibration specification. 


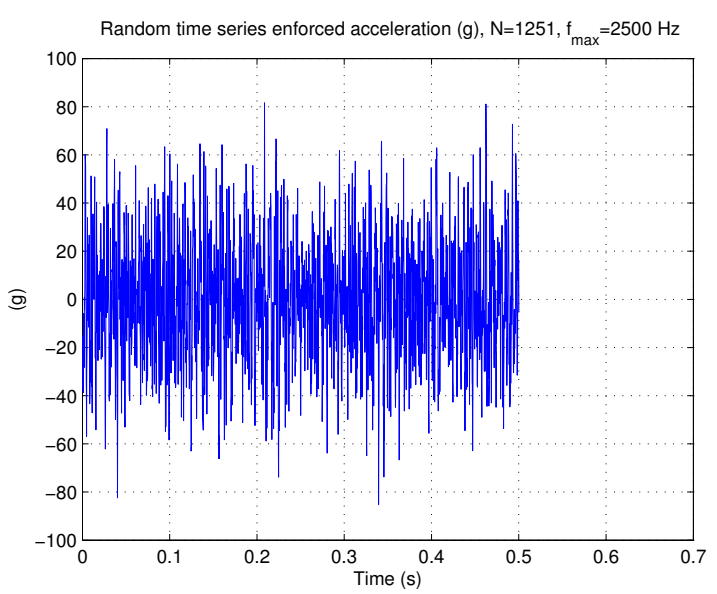

(a)

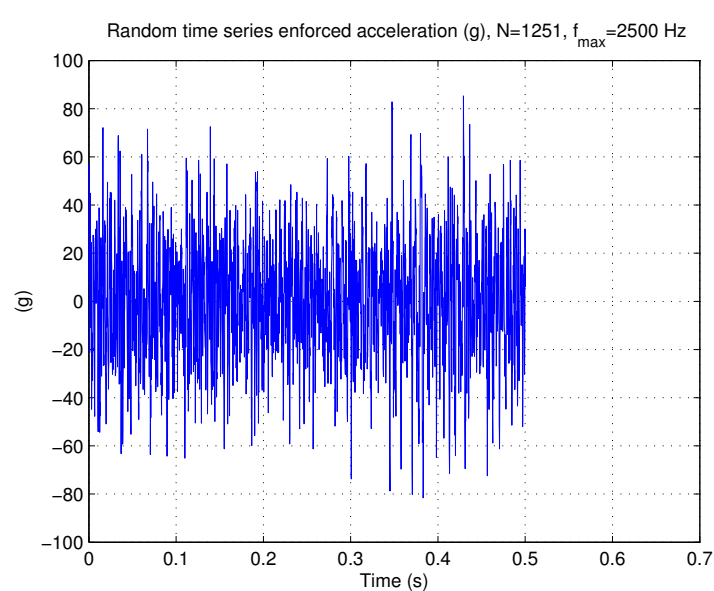

(b)

Figure 10: Synthesized time domain random accelerations, $\mu=0,1 \sigma=24.98 \mathrm{~g}, \lambda=0.01$, $\gamma=2.97(\lambda, \gamma$ mean values 10 samples $)[31]$

The SRS and ERS are divided into $N_{\text {field }}=100$ fields. For each field the maximum value of the SRS and ERS is detected and applied to synthesize the reduced number of data points of the SRS and the ERS into PSD of the random acceleration vibration specification using (11) and (10), respectively. Both random acceleration vibration specifications are shown in Fig. 12 (b). Further, the synthesized random acceleration vibration specifications $W_{S R S}$ and $W_{E R S}$ are compared to the original response spectrum of accelerometer Acc-4Y in Fig. 12(c). The

Table 1: Synthesis random acceleration vibration specification from ERS and SRS

\begin{tabular}{lccc}
\hline & \multicolumn{3}{c}{$Q$} \\
\cline { 2 - 4 } & 10 & 25 & 50 \\
\hline$W_{E R S}$ r.m.s. & 26.1 & 26.3 & 26.9 \\
$W_{S R S}$ r.m.s. $\mu$ & 24.0 & 23.1 & 22.5 \\
$W_{S R S}$ r.m.s. $\sigma$ & 0.8 & 0.6 & 0.4 \\
\hline Acc-4Y & \multicolumn{3}{c}{$25.0 \mathrm{G}_{r m s}$} \\
\hline
\end{tabular}

PSD spectra $W_{S R S}$ and $W_{E R S}$ are computed varying the amplification factor $Q=10,25,50$. The synthesized random accelerations are calculated from the original spectrum as shown in Fig 9, although, mean and standard deviation remain the same, but the distribution of the peaks alters in time, due to random arguments in the sine waves representation of the original PSD spectrum. That means that the SRS and $W_{S R S}$ are more or less random. Therefor the mean and standard deviation of $W_{S R S}$ is calculated from 10 samples. The PSD spectrum $W_{E R S}$ will not change. The r.m.s. values of the PSD spectra $W_{S R S}$ and $W_{E R S}$ are given in Table 1 . The synthesized r.m.s. values of PSD spectra of $W_{S R S}$ under estimate the r.m.s. value of the original PSD spectrum and the synthesized r.m.s. values of the PSD spectrum of $W_{E R S}$ show a higher r.m.s. values compared to the original PSD spectrum of the accelerometer Acc-4Y.

A further simplification of the random acceleration vibration spectra $W_{S R S}$ and $W_{E R S}$ can be achieved dividing the SRS and ERS spectra into less fields, however, this will result in higher r.m.s. values of the equivalent random acceleration vibration specifications. 


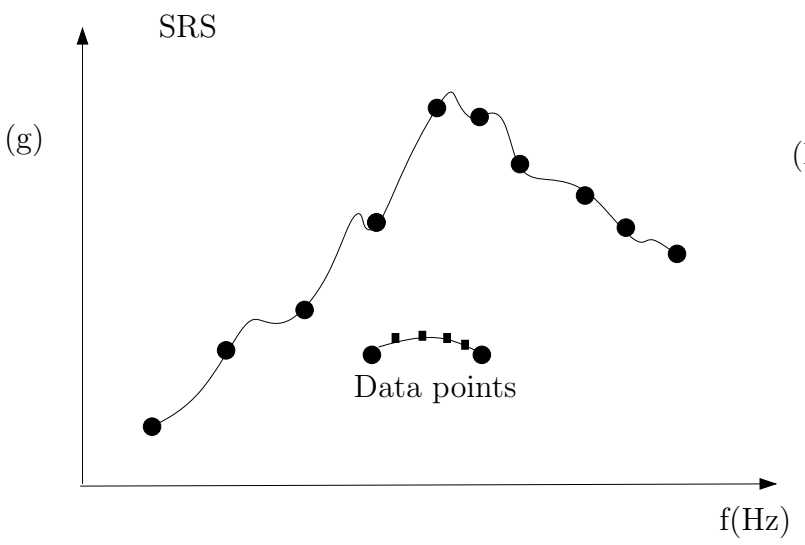

(a)

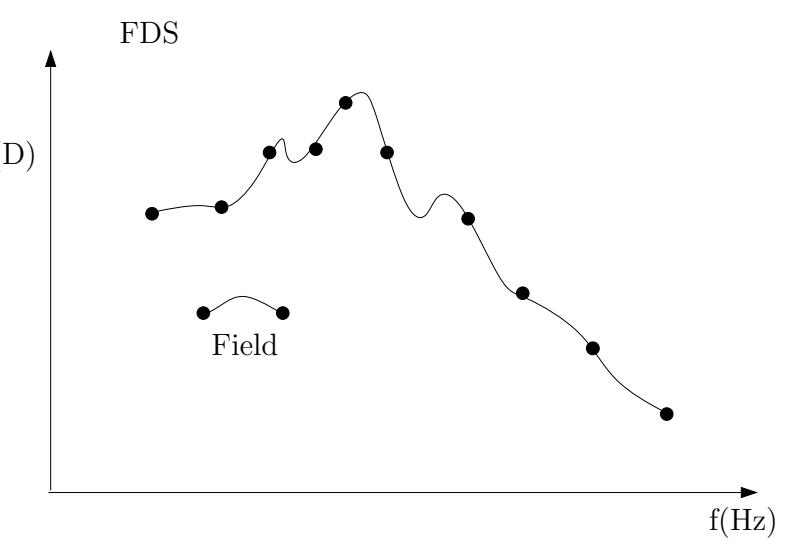

(b)

Figure 11: Division of spectra in fields

In Fig. 12 (d) the equivalent ERS from the equivalent $W_{E R S}$ is computed for $Q=10$ and compared to the original ERS.

\subsection{Synthesis of Random Acceleration Vibration Specification from FDS $_{d}$}

The $\mathrm{FDS}_{d}$ spectrum is computed both in the frequency and time domain. In the time domain the rain flow counting method (RF), as provided in Matlab ${ }^{\circledR}$, is used. The number of data points of the $\mathrm{FDS}_{d}$ in the frequency domain is 2481 and in the time domain 1251 . The number of data point in the original spectrum is namely 1251 too. The $\mathrm{FDS}_{d}$ is computed varying the amplification factor $Q=10,25,50$ and the fatigue exponent $b=4,8,12$. For $Q=10$ and $b=8$ the $\mathrm{FDS}_{d}$ are shown in Fig. 13(a). To compute the equivalent random acceleration vibration specifications from the $\mathrm{FDS}_{d}$, the spectra are divided into $N_{\text {field }}=100$ fields. $W_{\text {Freq }}$ is the synthesis of the random acceleration vibration specification taken from the $\mathrm{FDS}_{d}$ spectrum and $W_{R F}$ is the synthesis of $\operatorname{FDS}_{d, R F}$. For $Q=10$ and $b=8$ both synthesized FDS are shown in Fig. 13 (b). In Fig. 13(c) both synthesized random acceleration vibration specifications are compared to the random acceleration spectrum of Accelerometer Acc-4Y.

All computed data are given in Table 2. The mean values and the standard deviations of the $W_{R F}$ PSD spectra are calculated using 10 samples. The figures in between the brackets are the standard deviations.

Again it is noticed that the synthesized random acceleration vibration specifications $W_{R F}$ (time domain) are below the original spectrum of accelerometer Acc-4Y.

Table 2: Synthesis random acceleration vibration specification from $\mathrm{FDS}_{d}$

\begin{tabular}{lcccccc}
\hline & \multicolumn{6}{c}{$b$} \\
\cline { 2 - 7 } Q / r.m.s. & $W_{\text {Freq }}$ & $W_{R F}\left(\mathrm{RF}^{*}\right)$ & $W_{\text {Freq }}$ & $W_{R F}$ & $W_{F r e q}$ & $W_{R F}$ \\
\hline 10 & 26.1 & $24.0(0.3)$ & 26.2 & $23.8(0.5)$ & 26.2 & $23.1(0.4)$ \\
25 & 26.2 & $24.2(0.4)$ & 26.7 & $23.9(0.4)$ & 26.7 & $22.9(0.5)$ \\
50 & 27.0 & $24.4(0.4)$ & 27.4 & $24.0(0.4)$ & 27.4 & $22.8(0.5)$ \\
\hline Acc-4Y & \multicolumn{7}{c}{$25.0 \mathrm{G}_{r m s}$} \\
\hline * RF is Rain flow counting
\end{tabular}




\subsection{Synthesis of Random Acceleration Vibration Specification from FDS F $_{p}$}

The $\mathrm{FDS}_{p v}$ is quite similar to the $\mathrm{FDS}_{d}$. The $\mathrm{FDS}_{p v}$ spectrum is computed both in the frequency and time domain. In the time domain the rain flow counting method (RF) was used. The number of data points of the $\mathrm{FDS}_{p v}$ in the frequency domain is 2481 and in the time domain 1251. The $\mathrm{FDS}_{p v}$ is computed varying the amplification factor $Q=10,25,50$ and the fatigue exponent $b=4,8,12$. For $Q=10$ and $b=8$ the $\operatorname{FDS}_{p v}$ are shown in Fig. 14(a). To compute the synthesized random acceleration vibration specifications the $\mathrm{FDS}_{p v}$ spectra are divided in $N_{\text {field }}=100$ fields. $W_{\text {Freq }}$ is the synthesis of the random acceleration vibration specification taken from the $\mathrm{FDS}_{p v}$ spectrum and $W_{R F}$ is the synthesis of $\operatorname{FDS}_{p v, R F}$. For $Q=10$ and $b=8$ both synthesized FDS are shown in Fig. 14(b). In Fig. 14(c) both synthesized random acceleration vibration specifications are compared to the random acceleration spectrum of Acc-4Y accelerometer.

All computed data are given in Table 3. The mean values and the standard deviations of the $W_{R F}$ PSD spectra are calculated using 10 samples. The figures in between the brackets are the standard deviations.

It is again noticed that the r.m.s. values of the synthesized random acceleration vibration specifications $W_{R F}$ (time domain) are below the r.m.s. value of the original spectrum of accelerometer Acc- 4 Y.

Table 3: Synthesis random acceleration vibration specification from $\operatorname{FDS}_{p v}$

\begin{tabular}{lcccccc}
\hline & \multicolumn{5}{c}{$b$} \\
\cline { 2 - 7 } Q / r.m.s. & $W_{F r e q}$ & $W_{R F}\left(\mathrm{RF}^{*}\right)$ & $W_{\text {Freq }}$ & $W_{R F}$ & $W_{\text {Freq }}$ & $W_{R F}$ \\
\hline 10 & 26.1 & $23.9(0.2)$ & 26.1 & $24.1(0.4)$ & 26.1 & $23.2(0.5)$ \\
25 & 26.4 & $24.3(0.5)$ & 26.4 & $24.3(0.4)$ & 26.4 & $23.3(0.3)$ \\
50 & 27.5 & $25.0(0.6)$ & 27.5 & $24.5(0.4)$ & 27.5 & $23.2(0.4)$ \\
\hline Acc-4Y & \multicolumn{5}{c}{$25.0 \mathrm{G}_{r m s}$} \\
\hline * RF is Rain flow counting
\end{tabular}

\subsection{Synthesis of Equivalent Random Acceleration Vibration Specification based on Rayleigh Distribution of Peaks}

Table 4: Equivalent random acceleration vibration specification from Rayleigh distribution of peaks

\begin{tabular}{lccc}
\hline & \multicolumn{3}{c}{$Q$} \\
\cline { 2 - 4 }$W_{D P}$ & 10 & 25 & 50 \\
\hline Mean r.m.s. $\mu$ & 26.0 & 25.4 & 24.8 \\
Std-dev r.m.s. $\sigma$ & 0.7 & 0.4 & 0.2 \\
\hline Acc-4Y & \multicolumn{3}{c}{$25.0 \mathrm{G}_{r m s}$} \\
\hline
\end{tabular}

The analysis of the Rayleigh distribution of peaks (damage potential) is completely done in the time domain on a transient random acceleration as illustrated in Fig. 10. The rain flow method as provided by Matlab ${ }^{\mathbb{R}}$, is used to extract from the transient signal the amplitudes and corresponding number of cycles. The minimum value $A_{\min }$ of the distribution of 
peaks is used to calculate the number of positive crossings $n_{0}$ through $A_{\text {min }}$. The total time $T_{A>A_{\min }}=n_{o} / f_{i}, i=1,2, \cdots$, where $f_{i}$ is one the frequency in the range of the spectrum $(20-2500 \mathrm{~Hz})$. The number of crossings (spectrum of crossings) $n_{0}$ is shown in Fig. 15(a). In the application the total time is $T=0.5004 \mathrm{~s}$, in fact the duration of the random transient signal. The total equivalent random acceleration vibration spectrum is computed from (33). The random acceleration vibration spectrum is divided in $N_{\text {field }}=100$ fields, and maximum values of that spectrum in these fields and associated frequencies are indicated and applied to generate the reduced equivalent random acceleration vibration specification $W_{D P}$. The full and reduced synthesized equivalent random acceleration spectra are shown in Fig. 15(b). The comparison to the original random acceleration vibration spectrum of accelerometer Acc-4Y is provided in Fig. 15(c). The presentation of Figures 15 are based on an amplification factor $Q=10$.

The amplification factors are varied $Q=10,25,50$ and the results of the calculation are presented in Table 4. The mean values and standard deviations of the r.m.s values of $W_{\text {test }}$ are calculated from 10 samples.

\subsection{All equivalent random acceleration vibration specifications in one plot}

All synthesized equivalent random acceleration vibration specification from SRS, ESR, FDS , $_{\text {, }}$ $\mathrm{FDS}_{p v}$ and from the damage potential (Rayleigh distribution of peaks) are depicted in Fig 16 (a) and (b). It can be seen that all equivalent random acceleration specifications have the same shape and about the same r.m.s values of the spectra, however, the r.m.s. values of the equivalent spectra computed from the frequency domain are somewhat higher than calculated in the time domain.

\subsection{Influence Number of Fields $N_{\text {field }}$}

The number of fields $N_{\text {field }}$ defines to a high extent the details kept in the equivalent random acceleration vibration specification, although in combination with the number of available data points in the response spectra. In previous sections the computations of the equivalent vibration spectra was based on a number of fields $N_{\text {field }}=100$. The original random acceleration spectra of accelerometer Acc-4Y (Fig. 9) is rather smoothened in the lower frequency range, but in the higher frequencies the spectra still exhibit a number of peaks and valleys (i.e Fig 12). If we decrease the number of fields $N_{\text {field }}=50,25,10,5$, the equivalent random acceleration vibration specification becomes more smoothened, however, the r.m.s. value will increase, and at the other hand the vibration specification becomes more severe. This will be illustrated by an example computing the equivalent acceleration vibration specification from the original ERS assuming an amplification factor $Q=10$. The figures are shown in Table 5. The mean and standard deviation of the equivalent vibration spectrum $W_{S R S}$ is computed using 10 samples. If the number of fields $N_{\text {field }}<10$, then it becomes difficult to get an envelope of the response

Table 5: Equivalent random acceleration vibration spectra (r.m.s.) from ERS, SRS with varying $N_{\text {field }}$.

\begin{tabular}{lccccc}
\hline & \multicolumn{5}{c}{$N_{\text {field }}, Q=10$} \\
\cline { 2 - 6 } & 100 & 50 & 25 & 10 & 5 \\
\hline $\mathrm{W}_{\text {ERS }}$ r.m.s. & 26.1 & 27.2 & 28.0 & 30.9 & 23.5 \\
$\mathrm{~W}_{S R S}$ r.m.s. $\mu$ & 24.0 & 25.0 & 26.0 & 27.8 & 26.6 \\
$\mathrm{~W}_{S R S}$ r.m.s. $\sigma$ & 0.8 & 0.9 & 0.8 & 1.1 & 1.6 \\
\hline
\end{tabular}


spectra and a r.m.s. value of the equivalent random acceleration spectrum below the r.m.s. value of the original spectrum (Fig. 9) will be achieved. It is recommended to keep $N_{\text {field }}=$ $10-20$ as a minimum. In Fig. 17(a) the synthesized equivalent random acceleration vibration specifications, based on the ERS, SRS and $N_{\text {field }}=10$ are shown.

\section{CONCLUSIONS}

In this paper a number of damage spectra are discussed to characterize measured or computed random acceleration vibration spectra, most times defined in PSD $\left(\mathrm{g}^{2} / \mathrm{Hz}\right)$. The characterization is based on equivalent damage caused by extreme peaks (SRS, ERS, VRS and Rayleigh distribution of peaks or cumulative damage (FDS), using relative displacements and pseudo-velocities. The response spectra are all based on the response of SDOF systems exited to random accelerations, both in the time and frequency domain. The principles to compute the response spectra are illustrated in the Figures 2, 3, 4, and 5 .

For random acceleration vibration it is shown numerically, that the ERS is the same as the SRS (i.e. Fig. 12(a)). The ERS is calculated in the frequency domain, but the SRS is calculated in the time domain, therefor the computation of the ERS is more straight forward then the calculation of the SRS. Nowadays in spacecraft structure engineering practice the VRS is applied to compare to the SRS, however, it is recommended to use the ERS instead of the VRS., because the ERS match better with the SRS then the VRS.

The $\mathrm{FDS}_{d}$ and $\mathrm{FDS}_{p v}$ spectra are quite the same.

Miles' equation fulfills a key role in the synthesis process to generate equivalent random acceleration vibration specifications, however, this equation represents the r.m.s. acceleration response of a SDOF system excited at the base by white noise random acceleration, which is an idealization of the real random acceleration spectrum. The application of Miles' equation in the synthesis process is an approximation!

An envelope of the damage response spectrum is achieved by dividing the spectrum into a number of fields $N_{\text {field }}$. The lower $N_{\text {field }}$ to more severe and smoother the equivalent random acceleration vibration specification becomes. This process is shown in Fig. 11 .

All damage response spectra result in about the same equivalent random acceleration vibration specifications (see Fig. 16.

The methods described in this paper are applied to a real life problem, the random acceleration spectrum (PSD) measured during an acoustic noise test of an accelerometer placed on the outside panel of the VAATMLDS bread board solar array wing.

To handle uncertainties in damping and material properties the amplification factor $Q$ and the Basquin's exponent $b$ in the s-N curve have to be varied, in general, $Q=10,25,50$ and $b=4,8,12$. The worst case equivalent random acceleration vibration specification shall be selected, however, engineering judgement of the engineer is still needed.

The synthesized random transient signal derived from the Acc-4Y random spectrum has an invariant mean and standard deviation, however, the distribution of the peaks and the skewness and kurtosis show small variations.

\section{REFERENCES}

[1] Ahlin, K. (2006) Comparison of Test Specifications and Measured Field Data, Sound and Vibration, September, pages 22-24 
[2] Amzallag, C., Geray, J.P., Robert, J.L., Bahuaud, J. (1994) Standardization of Rain flow Counting Method for Fatigue Analysis, Fatigue, Vol 16, June, pages 287-293

[3] Benasciutti, D. (2004) Fatigue Analysis of Random Loadings, PhD University of Ferrara, Department of Engineering

[4] Biot, M.A. (1933) Theory of Elastic Systems Vibrating under Transient Impulse with an Application to Eartquake-Proof Buildings, Proceedings of the National Academy of Sciences, Vol. 19, No. 2, February, pages 261-268

[5] Biot, M.A. (1941) A Mechanical Analyzer for the Prediction of Earthquake Stresses, Bulletin of the Seismological Society of America, Vol. 31, No. 2, April, pages 150-171

[6] Crandall, S.H.(1962) Relation between Strain and Velocity in Resonant Vibration, Journal of Acoustical Society of America, Vol. 34(12), pages 160-1961

[7] Crandall, S.H., Mark, W.D. (1973) Random Vibration in Mechanical Systems, Academic Press

[8] DiMaggio, S.J., Sako, B.H. Rubin, S. (2003) Analysis of Non-stationary Vibroacoustic Flight Data using a Damage-Potential Basis, Journal of Spacecraft and Rockets, Vol. 40 No. 5, pages 682-689

[9] Dunham, W. (1999) Euler The Master of Us All, The Mathematical Society of America, ISBN 0-88385-328-0

[10] Eaton, R., Caro, E. (2012) Test Tailoring Methodology for Equipment Qualification, 2012 Spacecraft and launch Vehicle Dynamic Environments Workshop, June 19-21 El segundo, $\mathrm{Ca}$, USA, pages 37

[11] Girard, A., Imbert, J.F., Moreau, D. (1989) Derivation of European Satellite Equipment Test Specification from Vibro-Acoustic Test data, Acta Astronautica, Vol 10, No. 10, pages 797-803

[12] Halfpenny, A. (2006) Methods for Accelerating Dynamic Durability, 9th Int. Conf. Recent Advances in Structural Dynamics, Southampton, UK, pages 18

[13] Halfpenny, A., Walton, T.C. (2010) New Techniques for Vibration Qualification of Vibrating Equipment on Aircraft, Aircraft Airworthiness \& Sustainment 2010, pages 19

[14] Henderson, G.R., Piersol, A.G. (2003) Evaluating Vibration Environments Using the Shock Response Spectrum, Sound and Vibration, April, pages 18-20

[15] IABG, Space Division, Survey of Facilities, TN-TR-1000, Issue 12, may 2012

[16] Irvine, T. (2009) An Introduction to the Vibration Response Spectrum, Revision D, Vibrationdata, June 16, pages 20

[17] Irvine, T. (2012) Shock and Vibration Stress as a Function of Velocity, Revision C, Vibrationdata, May 21, pages 66 
[18] Irvine, T. (2012) An Alternate Damage Potential Method for Enveloping Nonstationary Random Vibration, 2012 Spacecraft and launch Vehicle Dynamic Environments Workshop, June 19-21 El segundo, Ca, USA, pages 28

[19] Kelly, R.D., Richman, G. (1969) Principles and Techniques of Shock Data Analysis, SVM5, The Shock and Vibration Information Center, US MOD

[20] Lalanne, C. (2002) Mechanical Vibration \& Shock, Fatigue Damage Volume V, HPS, ISBN $1-9039-9606-6$

[21] Lalanne, C. (2002) Mechanical Vibration \& Shock, Specification Development, Volume V, HPS, ISBN 1-9039-9607-4

[22] Madayag, A.F. (1969) Metal fatigue: Theory and Design, John Wiley \& Sons, ISBN 47156315-3

[23] McNeill, S.I. (2008) Implementing the Fatigue Damage Spectrum and Fatigue Equivalent Vibration Testing, Sound and Vibration, 79th Shock and Vibration Symposium, October 26-30, Orlando, Florida pages 1-20

[24] Miles, J.W. (1954), On structural fatigue under random loading, Journal of the Aeronautical Sciences, Vol. 21, 753-762

[25] Miles, R.N. (1992) Effect of Spectral Shape on Acoustic Fatigue Life Estimation, Journal of Sound and Vibration, Vol. 153 No. 2, pages 376-386

[26] Nieslony A. (2008) Determination of fragments of multi-axial service loading strongly influencing the fatigue of machine components, Mechanical Systems and Signal Processing, Vol. 23(8) pages. 2712-2721

[27] Ochi, M.K. (1981) Principles of Extreme ValuesStatistics and their Application, Paper presented at Extreme Loads response Analysis Symposium, Arlington, VA, October 1920, pages. $15-30$

[28] Preumont A. (1985) On the Peak Factor of Stationary Gaussian Processes, Journal of Sound and Vibration, 100(1), pages 15-34

[29] Shinozuka, M., Jan, C.M. (1972) Digital Simulation of Random Processes and Its Applications, Journal of Sound and Vibration , 25 (1), pages 111-128

[30] Smallwood, D.O. (1981) An Improved Recursive Formula for Calculating Shock Response Spectra, Shock and Vibration Bulletin, No. 51, pages 4-10

[31] Steinwolf, A., White, R.G. (1997) Probability Density Functions of Acoustically Induced Strains in Experiments with Composite Plates, AIAA Journal, Vol. 35 No. 12, pages 15831861

[32] Tủma, J., Koči, P. (2009) Calculation of Shock Response Spectrum, Colloquium 'Dynamics of Machines" 2009, Prague, February 3-4, pages 6

[33] Wijker, J.J. (2011) Final Presentation 'Vibro-Acoustic Analysis Test Methods for Large Deployable Structure' (VAATMLDS), ESA/TRP Study Contract AO/1-5659/08/NL/EM, Noordwijk, February 20, 2011. 
[34] Wijker, J.J. (2008) Spacecraft Structures, Springer, ISBN 978-3-540-75552

[35] Wijker, J.J. (2009) Random Vibrations in Spacecraft Structures Design, Springer, ISBN 978-90-2727-6

[36] Wirsching, P.H., Paez, T.L., Ortiz, H. (1995) Random Vibrations, Theory and Practice, John Wiley and Sons Inc., ISBN 0-471-58579-3

[37] Wood, W.L.. (1990) Practical Time-stepping Schemes, Clarendon Press, ISBN 0-19859677 


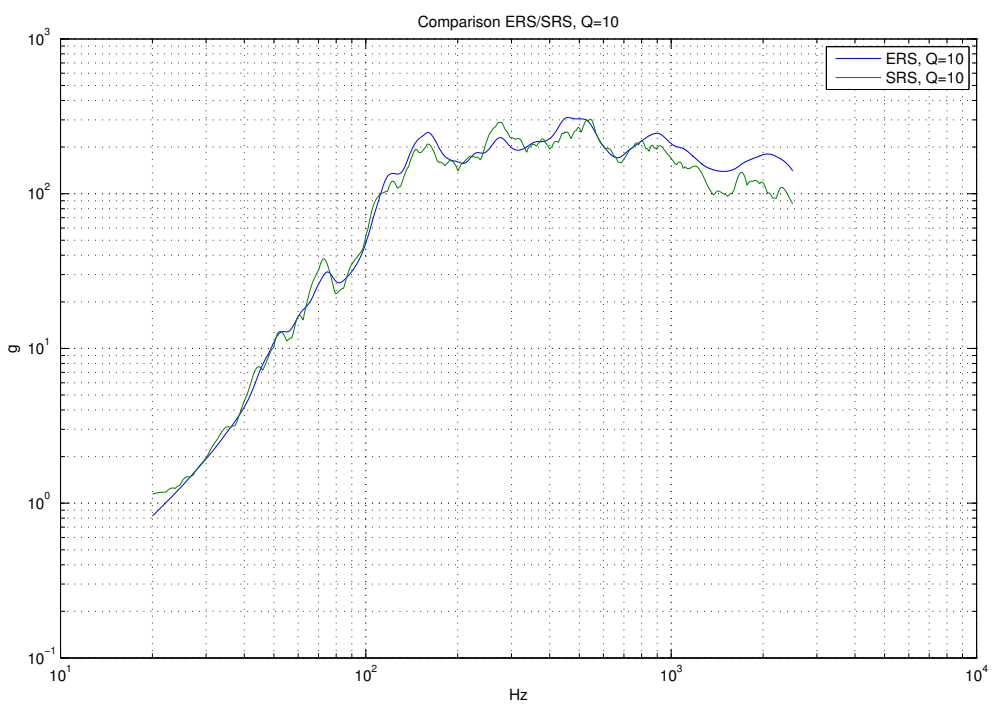

(a) SRS/ERS

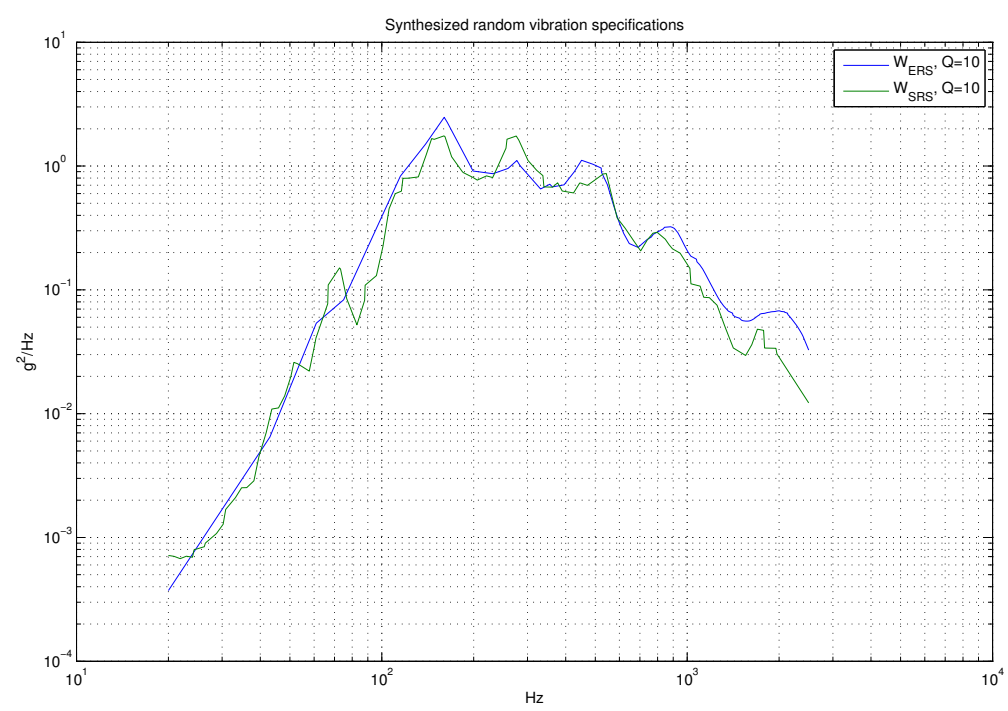

(b) $W_{S R S}, W_{E R S}$

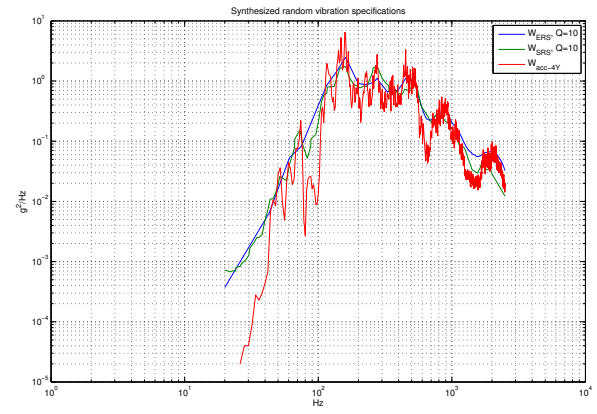

(c)

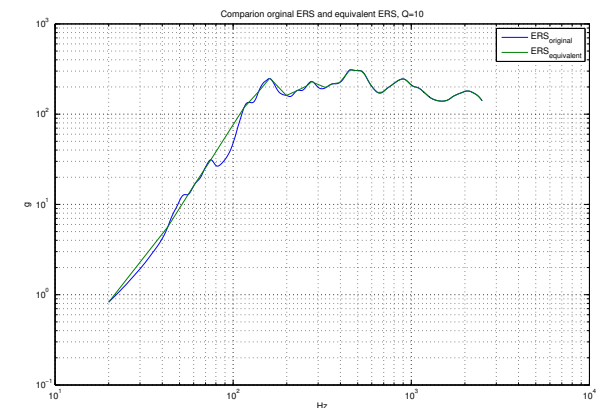

(d) ERS

Figure 12: Synthesis of equivalent random acceleration vibration specification from SRS/ERS 


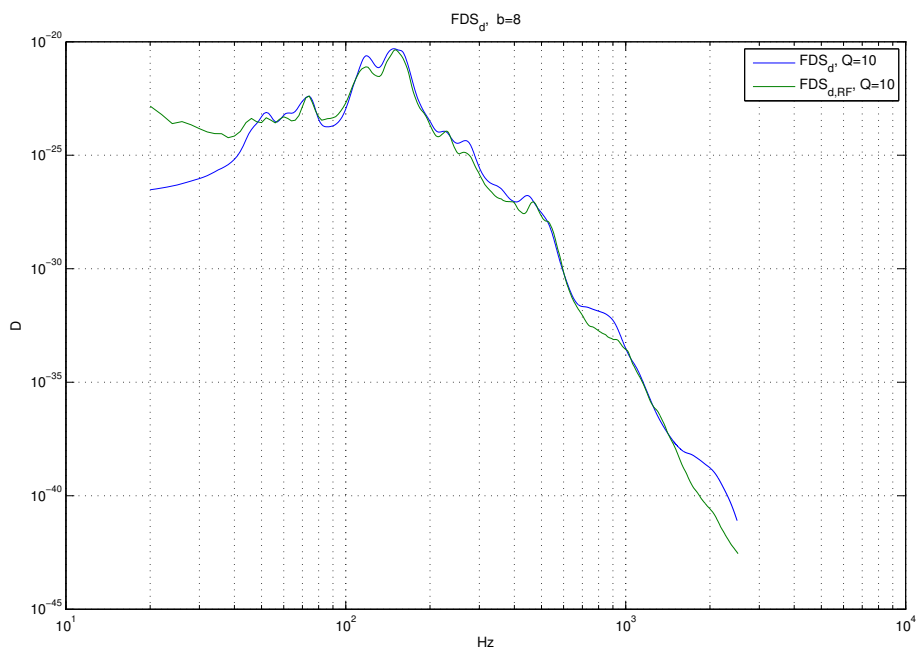

(a)

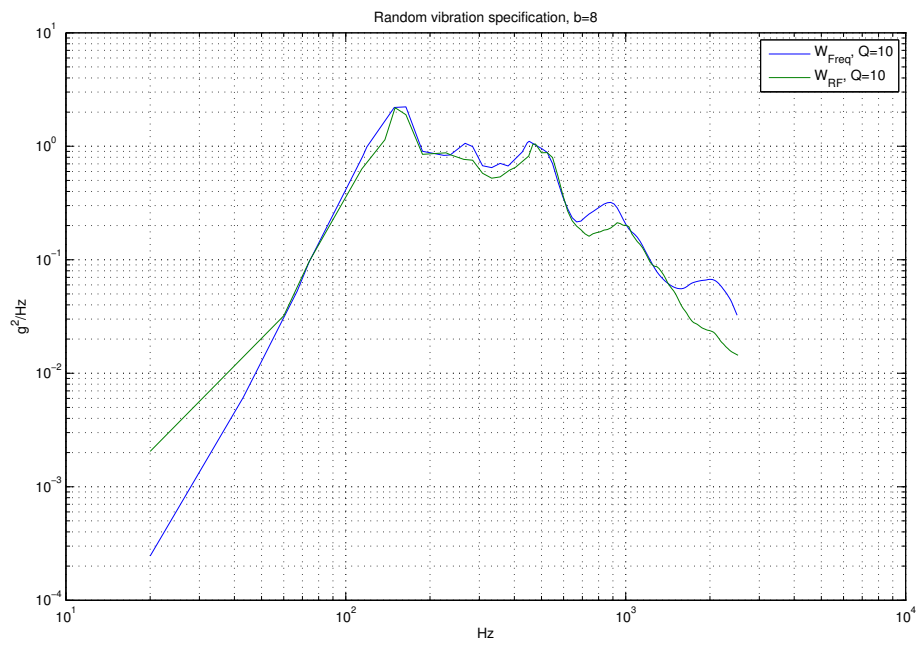

(b)

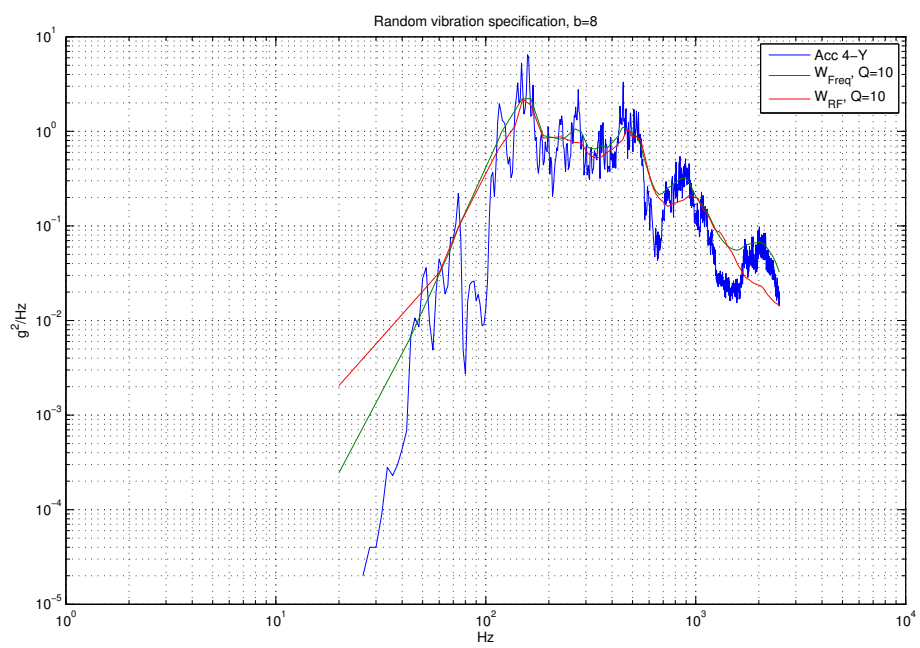

(c)

Figure 13: Synthesis of random acceleration vibration specification from $\mathrm{FDS}_{d}(\mathrm{RF}$ is rain flow counting) 


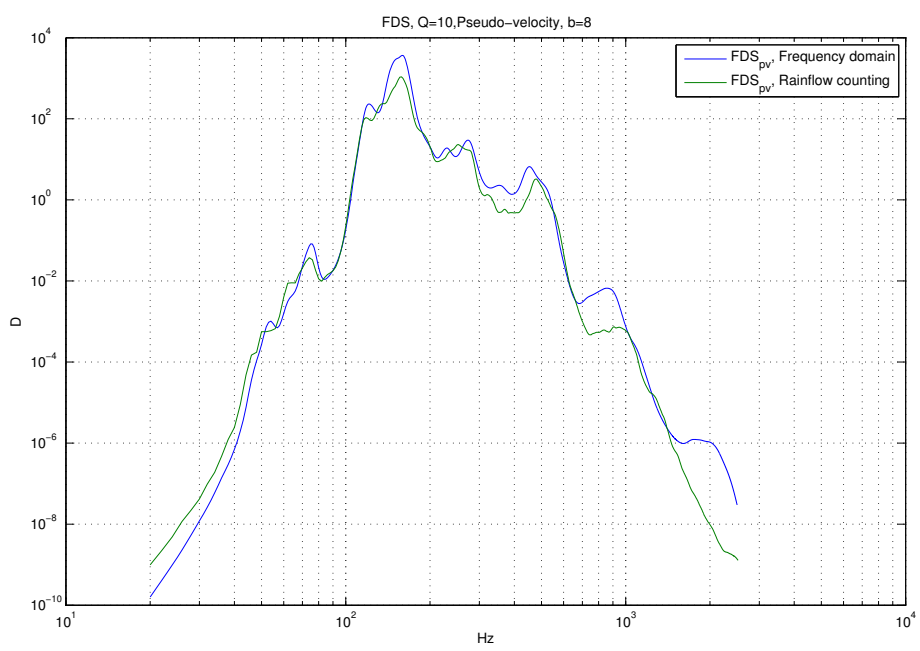

(a)

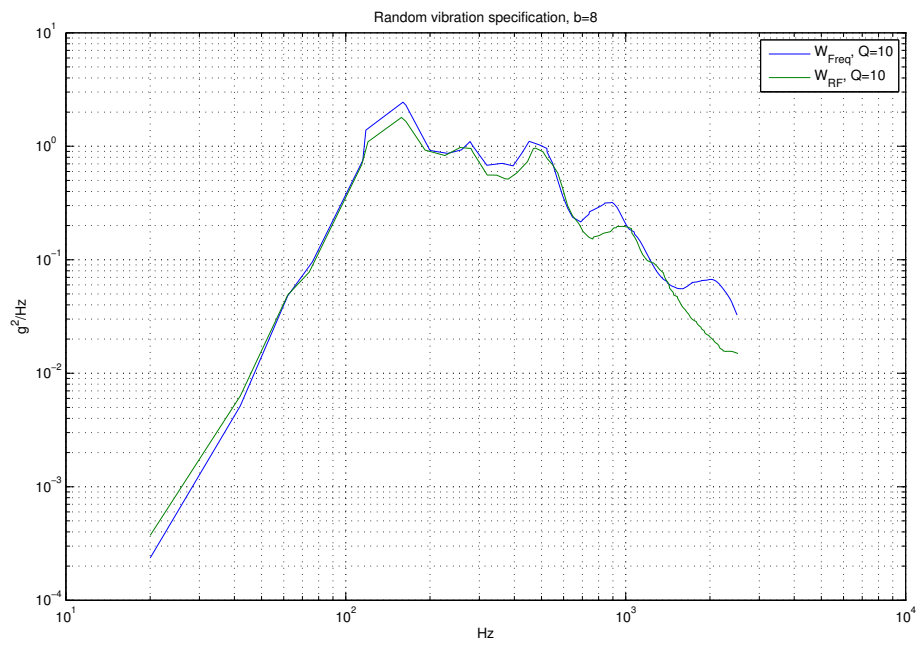

(b)

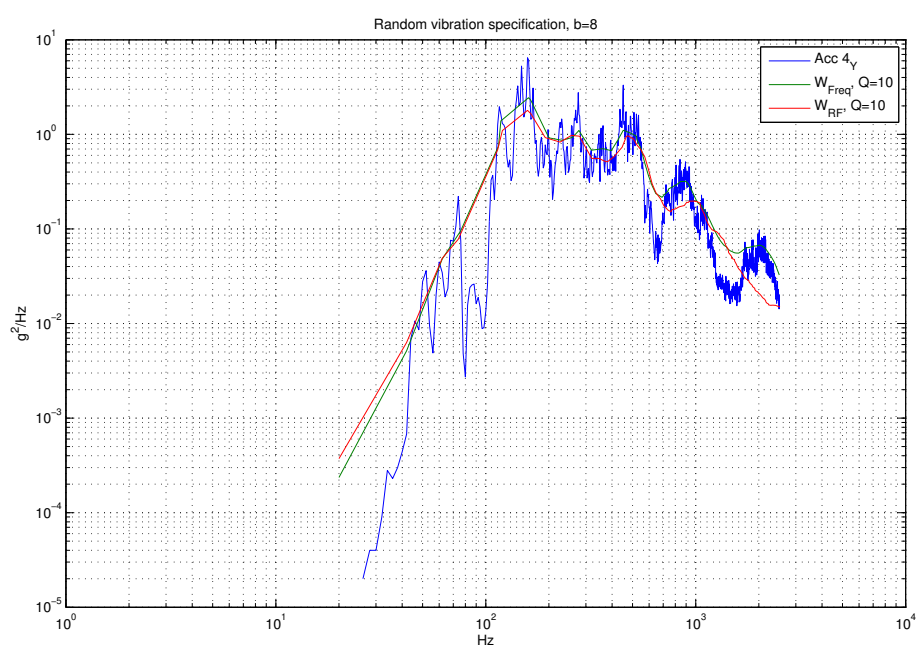

(c)

Figure 14: Synthesis of random acceleration vibration specification from $\mathrm{FDS}_{p v}(\mathrm{RF}$ is rain flow counting) 


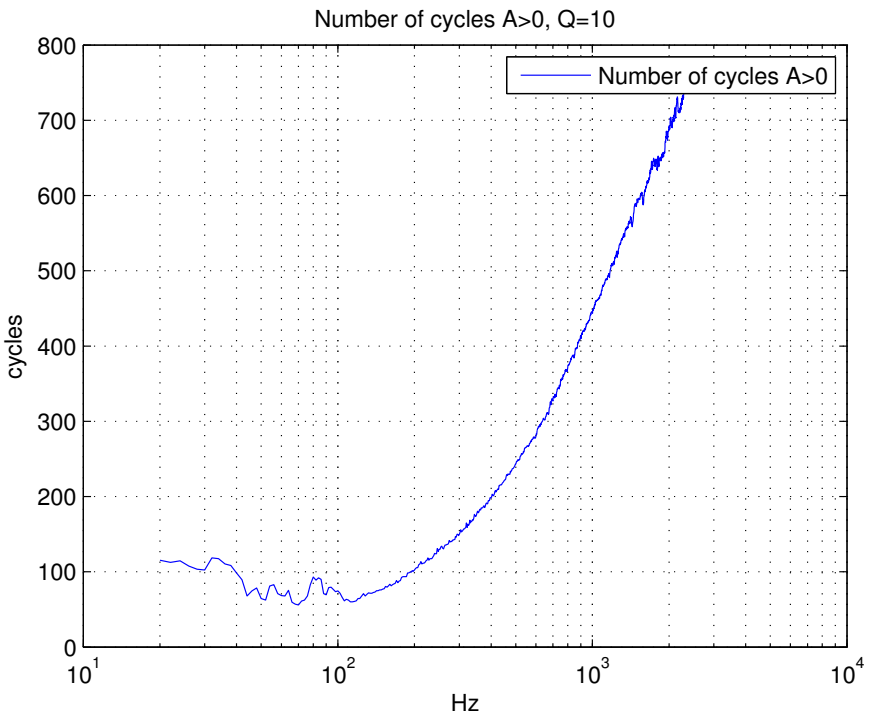

(a)

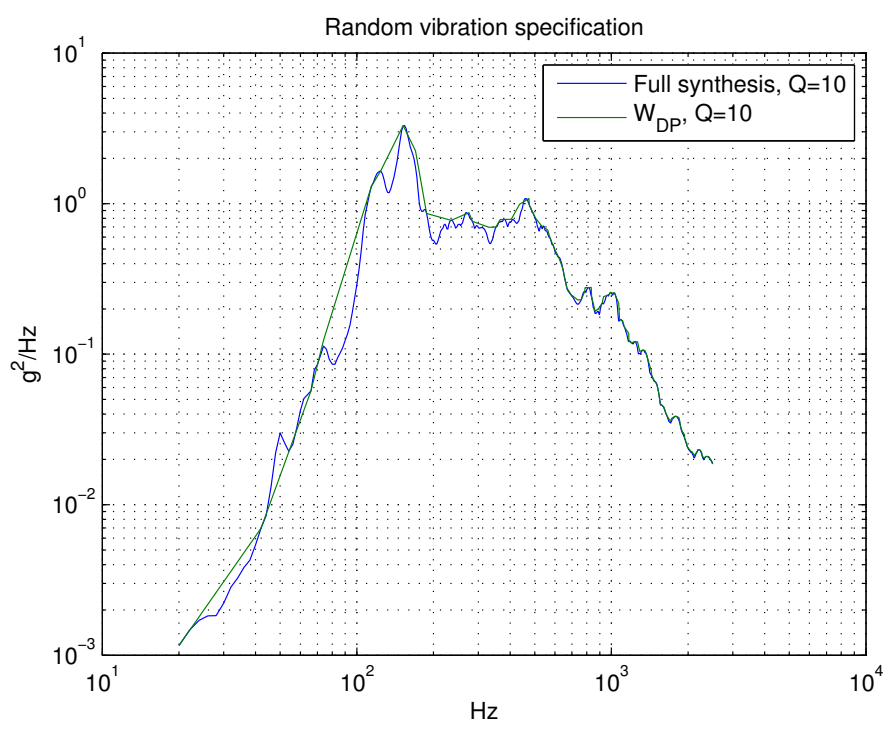

(b)

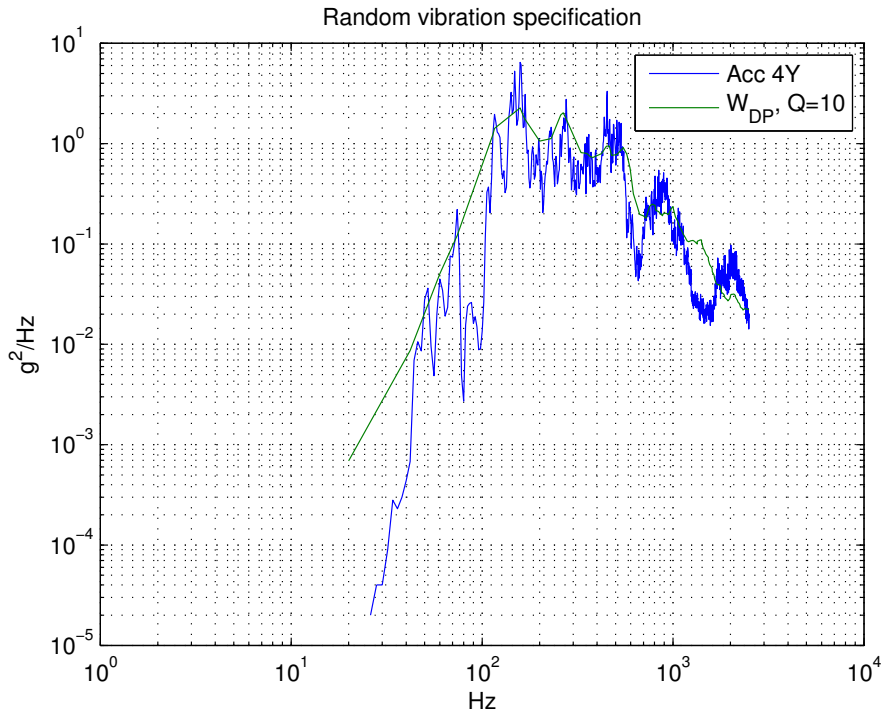

(c)

Figure 15: Synthesis of random acceleration vibration specification from potential damage (Rayleigh distribution of peaks) 


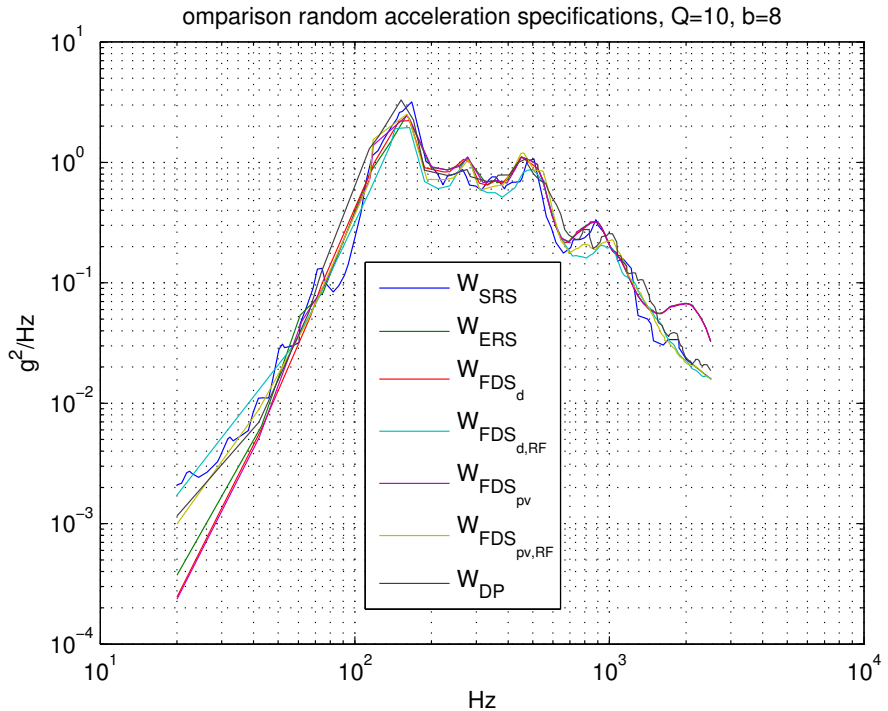

(a)

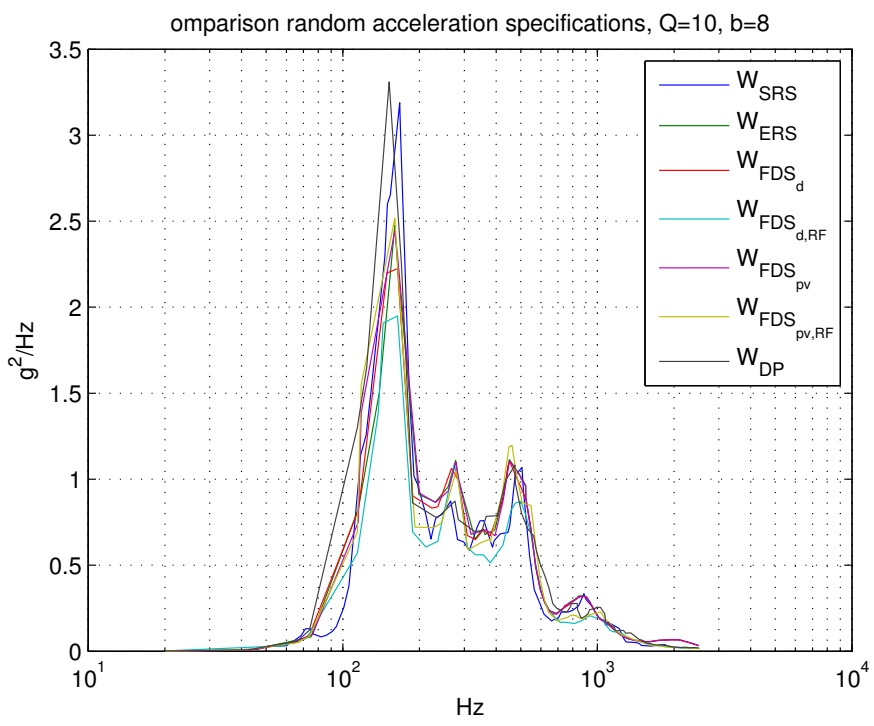

(b)

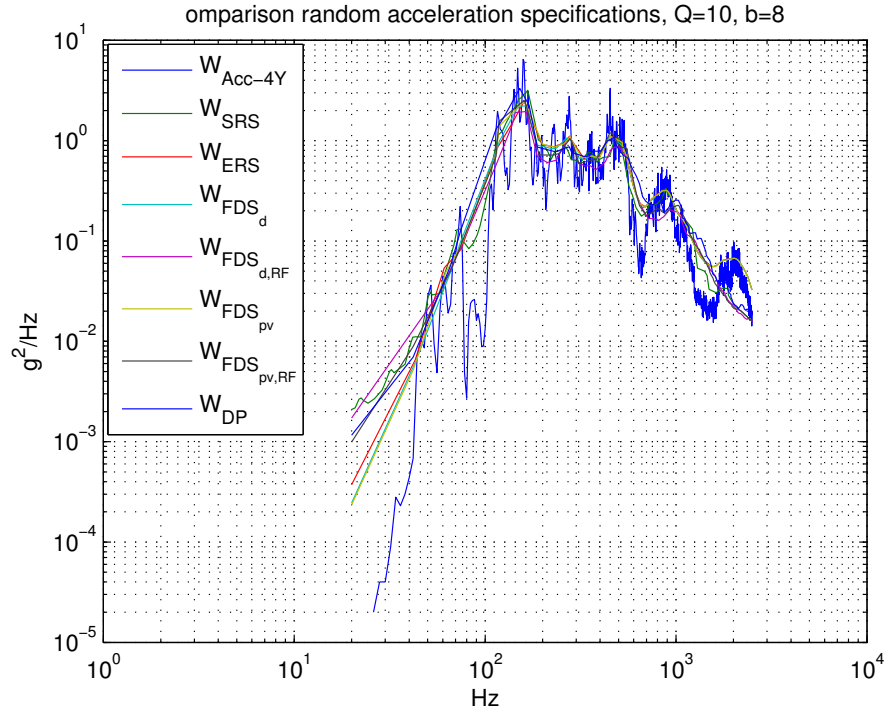

(c)

Figure 16: Presentation of all equivalent random acceleration vibration specifications 


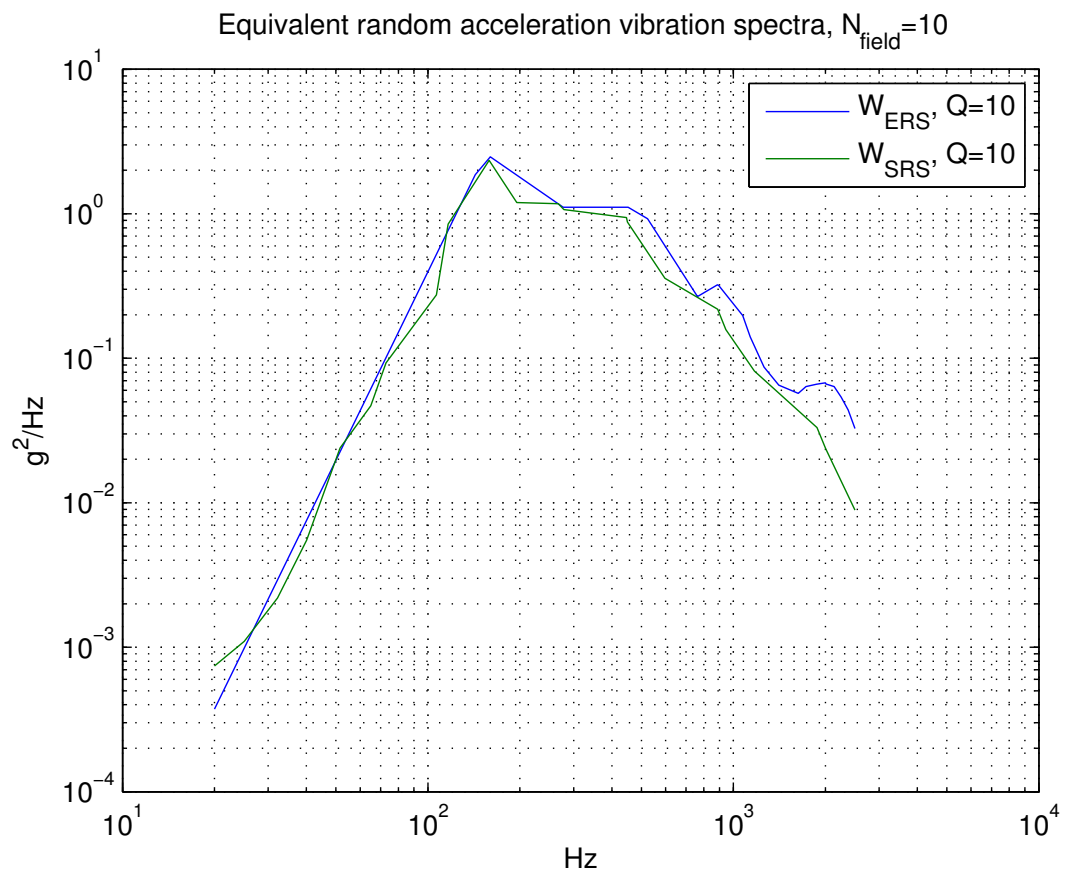

(a)

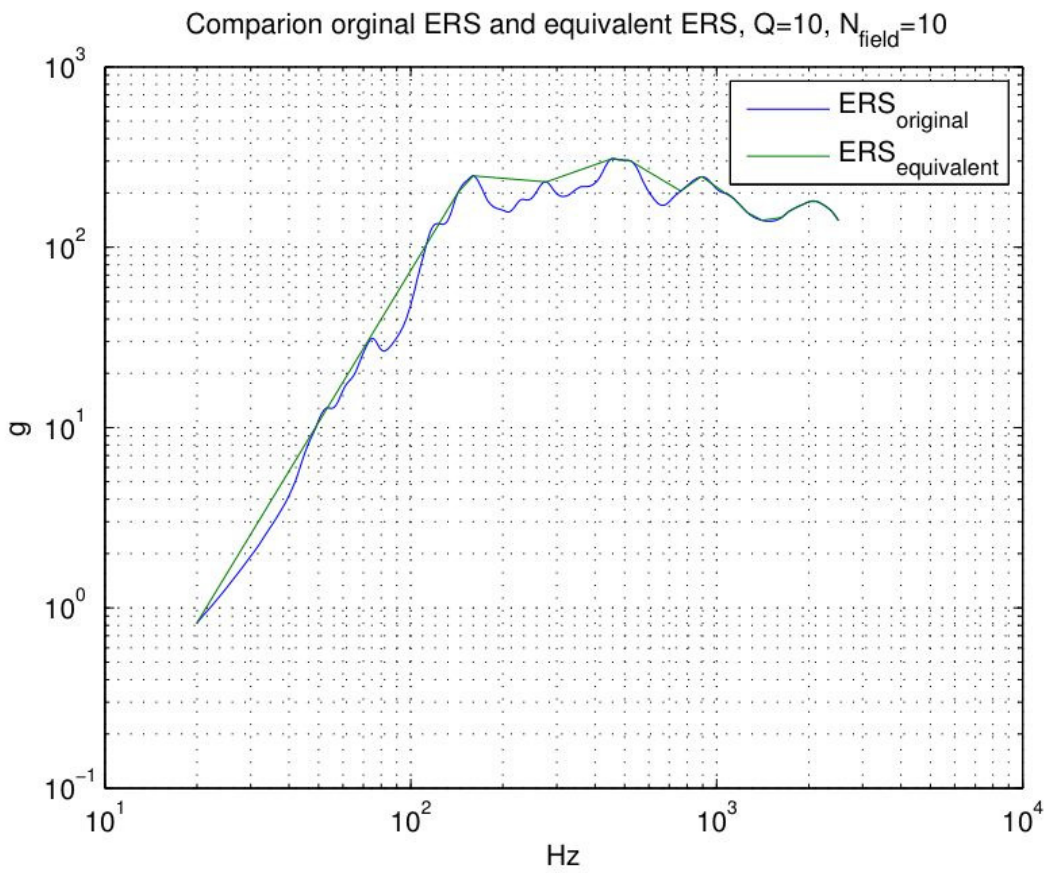

(b)

Figure 17: Equivalent random acceleration vibration spectra, $N_{\text {field }}=10$ 